\title{
Stability of hexagonal patterns in Bénard-Marangoni convection
}

\author{
B. Echebarria \\ Department of Engineering Sciences and Applied Mathematics, Northwestern University, 2145 Sheridan Road, Evanston, Illinois, 60208 \\ C. Pérez-García \\ Instituto de Física, Universidad de Navarra, E-31080 Pamplona, Navarra, Spain
}

(Received 24 August 2000; published 29 May 2001)

\begin{abstract}
Hexagonal patterns in Bénard-Marangoni (BM) convection are studied within the framework of amplitude equations. Near threshold they can be described with Ginzburg-Landau equations that include spatial quadratic terms. The planform selection problem between hexagons and rolls is investigated by explicitly calculating the coefficients of the Ginzburg-Landau equations in terms of the parameters of the fluid. The results are compared with previous studies and with recent experiments. In particular, steady hexagons that arise near onset can become unstable as a result of long-wave instabilities. Within weakly nonlinear theory, a two-dimensional phase equation for long-wave perturbations is derived. This equation allows us to find stability regions for hexagon patterns in BM convection.
\end{abstract}

DOI: 10.1103/PhysRevE.63.066307 PACS number(s): 47.54.+r, 47.27.Te, 47.20.Dr, 47.20.Ky

\section{INTRODUCTION}

Pattern formation in systems out of equilibrium has become an active area of research [1] since the pioneering work of Bénard a century ago [2]. He observed an array of hexagonal convective cells in a thin layer of spermaceti heated from below and open to the atmosphere. The liquid layer becomes unstable by the combined action of thermocapillary (Bénard effect) and buoyancy forces (Rayleigh effect), an instability currently known as Bénard-Marangoni (BM) convection. Later on the vast majority of studies, either theoretical or experimental, were made on buoyancy driven convection, which was named Rayleigh-Bénard $(R B)$ convection. Nevertheless, thermocapillary stresses may be important whenever a thermal gradient acts on a liquid-gas or a liquidliquid interface, especially in the case of thin layers, quite a common situation in many important technological processes [3]. BM convection intrinsically involves two fluids, but the gas can be considered as passive when dealing with a liquidgas interface. In these circumstances the theoretical description can be reduced to the usual one-fluid problem with a wave-number-dependent thermal exchange parameter (Biot number) [4]. From the experimental angle this approximation is ensured by thinning the gas gap between the liquid and a cover sapphire lid as much as possible [5-7]. Convective thresholds obtained using this coefficient fit the experimental values quite well and extend earlier theoretical results [8].

Several theoretical works have been devoted to the nonlinear analysis of BM convection [9-18]. The weakly nonlinear analysis, studying the relative stability of the different planforms, has been addressed both for pure Marangoni convection (no buoyancy) [11-13], as well as for the general BM case [15]. Some of these results are in good agreement with full numerical simulations of the basic hydrodynamic equations [14]. The general case, including the stability to arbitrary perturbations, was studied by Bestehorn [16]. However, an explicit derivation of the Ginzburg-Landau equations (GLE), including spatial terms, has only been done for pure Marangoni convection $[17,18]$. Here we extend these results to the case when both buoyancy and surface-tension effects are present. We will focus on the stability of hexagons as the different parameters in the fluid are varied. Stability analyses are performed by splitting perturbations in amplitude and phase components. We will show that the amplitude stability curves obtained previously in Refs. [12-15] are modified by the spatial terms. An explicit twodimensional (2D) phase equation can be derived analytically from the GLE [19-21], thus giving insight into the physical origin of long-wave instabilities for hexagons. In this paper we compute the coefficients in the phase equation, which allows us to complete the stability diagrams for BM convection.

The paper is organized as follows. In Sec. II we recall briefly the basic equations and boundary conditions (bc), and the linear stability analysis of BM convection. Section III is devoted to determining the amplitude equations. We first discuss the normal form with its coefficients, and, second, we calculate the linear and quadratic gradient terms. The amplitude instabilities are analyzed in Sec. IV. Section V discusses the phase equation and the stability regions for BM convection. Finally, Sec. VI contains a brief summary of the results and comparison with related work. For the sake of clarity, we have placed some specific calculations in two Appendices.

\section{EVOLUTION EQUATIONS FOR BM CONVECTION}

We consider a horizontal liquid layer of depth $d$ heated from below and open to the atmosphere, under a temperature difference $\Delta T$. In recent experiments a good thermal regulation is achieved by keeping the thin air layer in contact with a sapphire plate with a thermostatic bath [5-7]. From the difference between the heating plate and this bath, the temperature difference across the liquid layer $\Delta T$ can be inferred and the thermal exchanges between the liquid and the air quantified. Not far from threshold, air can be assumed to be a passive medium [4], so we can deal with the one-fluid 
model. Those experiments have been performed in convective cells with a horizontal length $L \gg d$, so that an infinitely extended layer can be assumed for calculations [1]. (Quantitative but not qualitative differences between theoretical and experimental results would appear for such big aspect ratios.)

Within the Boussinesq approximation and using the standard notation, the equations governing the problem expressed in rescaled nondimensional variables (obtained dividing space, time, velocity, and temperature by $d, d^{2} / \kappa$, $\kappa / d$, and $\Delta T$, respectively) read as

$$
\begin{gathered}
\operatorname{Pr}^{-1}\left[\frac{\partial \mathbf{v}}{\partial t}+(\mathbf{v} \cdot \boldsymbol{\nabla}) \mathbf{v}\right]=-\boldsymbol{\nabla} p+\operatorname{Ra} \theta \mathbf{e}_{z}+\nabla^{2} \mathbf{v}, \\
\frac{\partial \theta}{\partial t}+(\mathbf{v} \cdot \boldsymbol{\nabla}) \theta=\nabla^{2} \theta, \\
\boldsymbol{\nabla} \cdot \mathbf{v}=0
\end{gathered}
$$

where buoyancy effects are characterized by the Rayleigh number $\operatorname{Ra}=\alpha g d^{3} \Delta T / \nu \kappa$ and $\operatorname{Pr}=\nu / \kappa$ stands for the Prandtl number. For the usual silicon oils used in experiments $\operatorname{Pr} \approx 100$, so we can take $\operatorname{Pr}^{-1}=0$ as a reference value in the following. High Pr numbers mean that the dynamics is ruled by the temperature field and the mean flow effects are negligible.

Experiments on BM convection are most frequently performed with a rigid, heat-conducting lower plate (copper, aluminum, silicon). We will therefore consider the bc

$$
\mathbf{v}=\mathbf{0}, \quad T=T_{b}, \text { at } z=0 .
$$

The liquid-air interface is assumed to be undeformable, partially conducting and with a temperature-dependent surface tension:

$$
w=\partial_{z} \theta+\operatorname{Bi} \theta=\partial_{z}^{2} w+\operatorname{Ma} \nabla_{h}^{2} \theta=0, \text { at } z=1,
$$

where $\mathrm{Bi}$ (Biot number) accounts for the heat transfer through the interface. In general, $\mathrm{Bi}$ is a spatially dependent parameter but, for simplicity, we will take a constant reference value $\mathrm{Bi}=0.1$ typical in experiments. For very thin layers $(d<0.3 \mathrm{~mm})$ surface deformations can become important, giving rise to a longwavelength instability of the flat interface [22]. In the usual BM experiments, however, the thickness of the fluid layer is at least of the order of the millimeter and the surface deformation is negligible. Thermocapillary effects are quantified by means of the Marangoni number $\mathrm{Ma}=\gamma d \Delta T / \rho \nu \kappa$, related with Ra through a constant $\Gamma=\mathrm{Ma} / \mathrm{Ra}=\gamma / \rho \alpha g d^{2}$ that depends on the characteristics of the fluid and on the liquid depth $d$. The limit $\Gamma$ $\rightarrow 0$ corresponds to RB convection, while pure Marangoni convection $(\Gamma \rightarrow \infty)$ is reached when gravitational forces are absent or the layer thickness is very small $\left(\mathrm{Ra} \sim d^{2} \mathrm{Ma}\right)$. We are interested in studying the stability of hexagon patterns as this parameter $\Gamma$ is varied.

Below a critical value of the temperature difference across the layer, the fluid remains in a conductive state:

$$
\mathbf{v}_{\text {cond }}=\mathbf{0}, \quad T_{\text {cond }}=-z+T_{b} .
$$

Perturbations around this state $\mathbf{v}=\mathbf{v}, \theta=T-T_{\text {cond }}, \pi=p$ $-p_{\text {cond }}$ satisfy

$$
\begin{gathered}
\operatorname{Pr}^{-1}\left[\frac{\partial \mathbf{v}}{\partial t}+(\mathbf{v} \cdot \boldsymbol{\nabla}) \mathbf{v}\right]=-\boldsymbol{\nabla} \pi+\operatorname{Ra} \theta \mathbf{e}_{z}+\nabla^{2} \mathbf{v} \\
\frac{\partial \theta}{\partial t}+(\mathbf{v} \cdot \boldsymbol{\nabla}) \theta=w+\nabla^{2} \theta \\
\boldsymbol{\nabla} \cdot \mathbf{v}=\mathbf{0}
\end{gathered}
$$

with the boundary conditions (bc):

$$
\begin{gathered}
\mathbf{v}=0, \quad \theta=0, \quad \text { at } z=0, \\
\partial_{z}^{2} w+\mathrm{Ma} \nabla_{h}^{2} \theta=\partial_{z} \theta+\operatorname{Bi} \theta=w=0, \quad \text { at } z=1 .
\end{gathered}
$$

It is possible to write Eqs. (1)-(3) in a more compact form:

$$
\mathcal{L} f=\mathcal{N}(f),
$$

where $f=(\mathbf{v}, \theta, \pi)$ denotes the eigenfunctions and $\mathcal{L}$ the linear operator, defined as

$$
\mathcal{L}=\left(\begin{array}{ccc}
\boldsymbol{\nabla}^{2} & \operatorname{Ra}_{z} & -\boldsymbol{\nabla} \\
\mathbf{e}_{z} & \boldsymbol{\nabla}^{2} & 0 \\
\boldsymbol{\nabla} & 0 & 0
\end{array}\right)
$$

and $\mathcal{N}$ stands for the nonlinear terms:

$$
\mathcal{N}(f)=\left(\begin{array}{c}
\operatorname{Pr}^{-1}\left[\partial_{t} \mathbf{v}+(\mathbf{v} \cdot \boldsymbol{\nabla}) \mathbf{v}\right] \\
\partial_{t} \theta+(\mathbf{v} \cdot \boldsymbol{\nabla}) \theta \\
0
\end{array}\right) .
$$

\section{Linear stability analysis}

We use the growth rate as the eigenvalue for the linear operator [15], so that

$$
\left(\begin{array}{ccc}
\boldsymbol{\nabla}^{2} & \mathrm{Ra}_{\mathbf{z}} & -\boldsymbol{\nabla} \\
\mathbf{e}_{z} & \boldsymbol{\nabla}^{2} & 0 \\
\boldsymbol{\nabla} & 0 & 0
\end{array}\right)\left(\begin{array}{c}
\mathbf{v} \\
\theta \\
\boldsymbol{\pi}
\end{array}\right)=\sigma\left(\begin{array}{ccc}
\operatorname{Pr}^{-1} & 0 & 0 \\
0 & 1 & 0 \\
0 & 0 & 0
\end{array}\right)\left(\begin{array}{c}
\mathbf{v} \\
\theta \\
\boldsymbol{\pi}
\end{array}\right) .
$$

Solutions of these equations are expanded in terms of normal modes:

$$
\left(\mathbf{v}_{\gamma}, \theta_{\gamma}, \pi_{\gamma}\right)=\left[U_{\gamma}^{k}(z), V_{\gamma}^{k}(z), W_{\gamma}^{k}(z), \Theta_{\gamma}^{k}(z), \Pi_{\gamma}^{k}(z)\right] e^{i \mathbf{k} \cdot \mathbf{x}},
$$

where $W_{\gamma}^{k}(z)$ and $\Theta_{\gamma}^{k}(z)$ satisfy

$$
\begin{gathered}
\left(D^{2}-k^{2}\right)^{2} W_{\gamma}^{k}-\operatorname{Ra} k^{2} \Theta_{\gamma}^{k}=\operatorname{Pr}^{-1} \sigma_{\gamma}^{k}\left(D^{2}-k^{2}\right) W_{\gamma}^{k}, \\
\left(D^{2}-k^{2}\right) \Theta_{\gamma}^{k}+W_{\gamma}^{k}=\sigma_{\gamma}^{k} \Theta_{\gamma}^{k},
\end{gathered}
$$

with the bc

$$
W_{\gamma}^{k}(0)=D W_{\gamma}^{k}(0)=W_{\gamma}^{k}(1)=0,
$$


$\Theta_{\gamma}^{k}(0)=D \Theta_{\gamma}^{k}(1)+\operatorname{Bi} \Theta_{\gamma}^{k}(1)=D^{2} W_{\gamma}^{k}(1)+\operatorname{Ma} k^{2} \Theta_{\gamma}^{k}(1)=0$.

(Notice that the singular limit $k \rightarrow 0$ must be considered apart [15]. We present this case in Appendix A). For each value of $k$ there is a discrete set of vertical eigenfunctions that are indexed with the subscript $\gamma$. The condition $\sigma=0$ fixes the marginal curve $\mathrm{Ma}=\mathrm{Ma}(k ; \mathrm{Pr}, \mathrm{Bi}, \Gamma)$. The minimum of this curve gives the critical Marangoni and wave number $\left(\mathrm{Ma}_{c}, k_{c}\right)$ at the onset of convection. Let us recall that the critical values $\mathrm{Ra}_{c}$ and $\mathrm{Ma}_{c}$ lay approximately on the line $\mathrm{Ma}_{c} / \mathrm{Ma}_{0 c}+\mathrm{Ra}_{c} / \mathrm{Ra}_{0 c}=1$ (Nield's relation [8]) where $M_{0 c}$ $\left(\mathrm{Ra}_{0 c}\right)$ is the critical value of $\mathrm{Ma}(\mathrm{Ra})$ in the limit $\mathrm{Ra}=0$ $(\mathrm{Ma}=0)$. Typical values for small $\mathrm{Bi}$ are $\mathrm{Ma}_{0 c} \approx 80$ and $\mathrm{Ra}_{0 c} \approx 670$.

In the literature two main criteria have been used to discern whether buoyancy or thermocapillary effects are dominant: (a) $\mathrm{Ma}_{c}>\mathrm{Ra}_{c}$ [23], equivalent to $\Gamma \geqslant 1\left[\tan ^{-1}(\Gamma)\right.$ $\approx 45^{\circ}$, and (b) $\mathrm{Ma}_{c}>\left(\mathrm{Ma}_{0 c} / \mathrm{Ra}_{0 c}\right) \mathrm{Ra}_{c}$ [3], equivalent to $\Gamma$ $\geqslant \mathrm{Ma}_{0 c} / \mathrm{Ra}_{0 c} \approx 0.12\left[\tan ^{-1}(\Gamma) \approx 7^{\circ}\right]$, for $\mathrm{Bi}=0$. The first criterion is quite restrictive and should be considered as the lower limit of a Marangoni-dominated instability, while the second can be viewed as the upper limit under which the buoyancy effects are dominant. For typical liquids [24] $\Gamma$ $=0.08 d^{2}(\mathrm{~cm})$, so that the Marangoni effect is dominant for $d<3 \mathrm{~mm}$ and the buoyancy effect for $d>8 \mathrm{~mm}$.

Before closing this section let us mention that the linear operator $\mathcal{L}$ is not self-adjoint. Therefore, the adjoint must be calculated for a subsequent nonlinear analysis. It is defined by the relationship:

$$
\left\langle f^{*}, \mathcal{L}_{c} f\right\rangle=\left\langle f, \mathcal{L}_{c}^{*} f^{*}\right\rangle
$$

in which the scalar product \langle\rangle is defined by:

$$
\langle a, b\rangle=\int_{V} \bar{a} b d V=\lim _{L \rightarrow \infty} \frac{1}{4 L^{2}} \int_{-L}^{L} \int_{-L}^{L} \int_{0}^{1} \bar{a} b d z d x d y .
$$

The form of $\mathcal{L}^{*}$ and $f^{*}=\left(\mathbf{v}^{*}, \pi^{*}, \theta^{*}\right)$ are deduced in Appendix A.

\section{AMPLITUDE EQUATIONS FOR BM CONVECTION}

In this section we perform a weakly nonlinear analysis of the hexagon planform observed in BM experiments. We therefore examine the stability of a hexagon $\psi \propto A_{1} e^{i \mathbf{k}_{1} \cdot \mathbf{x}}$ $+A_{2} e^{i \mathbf{k}_{2} \cdot \mathbf{x}}+A_{3} e^{i \mathbf{k}_{3} \cdot \mathbf{x}}+$ c.c. made up of three modes linked by the resonance condition $\mathbf{k}_{1}+\mathbf{k}_{2}+\mathbf{k}_{3}=\mathbf{0}$. It is worth mentioning that symmetry arguments are sufficient to determine the normal form for the amplitudes $A_{i}$ [25]

$$
\tau_{0} \partial_{t} A_{1}=\epsilon A_{1}+\alpha \bar{A}_{2} \bar{A}_{3}-g_{1}\left|A_{1}\right|^{2} A_{1}-g_{2}\left(\left|A_{2}\right|^{2}+\left|A_{3}\right|^{2}\right) A_{1},
$$

in which $\epsilon$ stands for the distance to threshold (in our case $\left.\epsilon \equiv\left(M-M_{c}\right) / M_{c}=\left(R-R_{c}\right) / R_{c}\right)$ and the coefficients $\tau_{0}, \alpha, g_{1}$ and $g_{2}$ depend on the particular problem under consideration. (The equations for $A_{2}, A_{3}$ are obtained by cyclic permutation of the indices).

\section{A. Normal forms and amplitude equations}

The coefficients in Eq. (23) can be obtained from the basic hydrodynamic equations by means of a Galerkin expansion [15]. We recall briefly the main steps of the this technique. First Eq. (12) is projected over the adjoint modes, so the linear part becomes:

$$
\begin{aligned}
\left\langle\left(\mathbf{v}^{*}, \theta^{*}, \pi^{*}\right) \mathcal{L}\left(\begin{array}{c}
\mathbf{v} \\
\theta \\
\pi
\end{array}\right)\right\rangle & =\left\langle\left(\mathbf{v}^{*}, \theta^{*}, \pi^{*}\right) \sigma_{k}\left(\begin{array}{c}
\operatorname{Pr}^{-1} \mathbf{v} \\
\theta \\
0
\end{array}\right)\right\rangle \\
& =\sigma_{k}\left\langle\operatorname{Pr}^{-1} \mathbf{v}^{*} \cdot \mathbf{v}+\theta^{*} \theta\right\rangle
\end{aligned}
$$

and the nonlinear term gives

$$
\begin{aligned}
& \left\langle\left(\mathbf{v}^{*}, \theta^{*}, \pi^{*}\right)\left(\begin{array}{c}
\operatorname{Pr}^{-1}\left(\partial_{t} \mathbf{v}+(\mathbf{v} \cdot \boldsymbol{\nabla}) \mathbf{v}\right) \\
\partial_{t} \theta+(\mathbf{v} \cdot \boldsymbol{\nabla}) \theta \\
0
\end{array}\right)\right\rangle \\
& =\partial_{t}\left\langle\operatorname{Pr}^{-1} \mathbf{v}^{*} \cdot \mathbf{v}+\theta^{*} \theta\right\rangle+\operatorname{Pr}^{-1}\left\langle\mathbf{v}^{*} \cdot[(\mathbf{v} \cdot \boldsymbol{\nabla}) \mathbf{v}]\right\rangle \\
& \quad+\left\langle\theta^{*}(\mathbf{v} \cdot \boldsymbol{\nabla}) \theta\right\rangle .
\end{aligned}
$$

Second, the fields are expanded in series of the linear eigenfunctions $\left(\mathbf{v}_{\gamma}^{k_{i}}, \theta_{\gamma}^{k_{i}}\right)$ with time-dependent amplitude coefficients [26]

$$
\left(\begin{array}{c}
\mathbf{v} \\
\theta
\end{array}\right)=\sum_{i, \gamma} A_{\gamma}^{k_{i}}(t)\left(\begin{array}{c}
\mathbf{v}_{\gamma}^{k_{i}} \\
\theta_{\gamma}^{k_{i}}
\end{array}\right)
$$

Third, the vertical component and the wave numberdependent planar part are expanded separately in Eqs. (7)(9) and the following hierarchy of equations is obtained:

$$
\partial_{t} A_{\gamma}^{k_{i}}=\sigma_{\gamma}^{k_{i}} A_{\gamma}^{k_{i}}+\sum_{\nu \rho} \sum_{j l} \mathcal{B}_{\gamma \nu \rho}\left(k_{i}, k_{j}, k_{l}\right) A_{\nu}^{k_{j}} A_{\rho}^{k_{l}},
$$

where the coefficients $\mathcal{B}_{\gamma \nu \rho}\left(k_{i}, k_{j}, k_{l}\right)$ depend on the integrals:

$$
\begin{aligned}
& \mathcal{B}_{\gamma \nu \rho}\left(k_{i}, k_{j}, k_{l}\right)\left(\operatorname{Pr}^{-1}\left\langle\mathbf{v}_{\gamma}^{k_{i}^{*}} \cdot\left[\left(\mathbf{v}_{\nu}^{k_{j}} \cdot \nabla\right) \mathbf{v}_{\rho}^{k_{l}}\right]\right\rangle+\left\langle\theta_{\gamma}^{k_{i}^{*}}\left(\mathbf{v}_{\nu}^{k_{j}} \cdot \nabla\right) \theta_{\rho}^{k_{l}}\right\rangle\right) \\
&\left\langle\operatorname{Pr}^{-1} \mathbf{v}_{\gamma}^{k_{i}^{*}} \cdot \mathbf{v}_{\gamma}^{k_{i}}+\theta_{\gamma}^{k_{i}^{*}} \theta_{\gamma}^{k_{i}}\right\rangle
\end{aligned}
$$

(see Appendix B). Near onset the growth rate $\sigma$ can be expanded as $\sigma \simeq 0+(\partial \sigma / \partial \epsilon) \epsilon \equiv \tau_{0}^{-1} \epsilon$. (An explicit expression for $\tau_{0}$ can be found in Appendix A 3.)

Finally, let us mention that the Galerkin method becomes useful if the infinite set (27) can be truncated at a suitable order. The expansion (26) involves the marginal modes (drawn by full arrows in Fig. 1), as well as higher harmonics (dashed arrows in the same figure). The latter are damped modes $\left(\sigma\left(k^{u}\right) \ll 0\right)$ and can be eliminated adiabatically. Up to cubic order in the amplitude equations only the first harmonics of the marginal modes must be considered. Besides, 


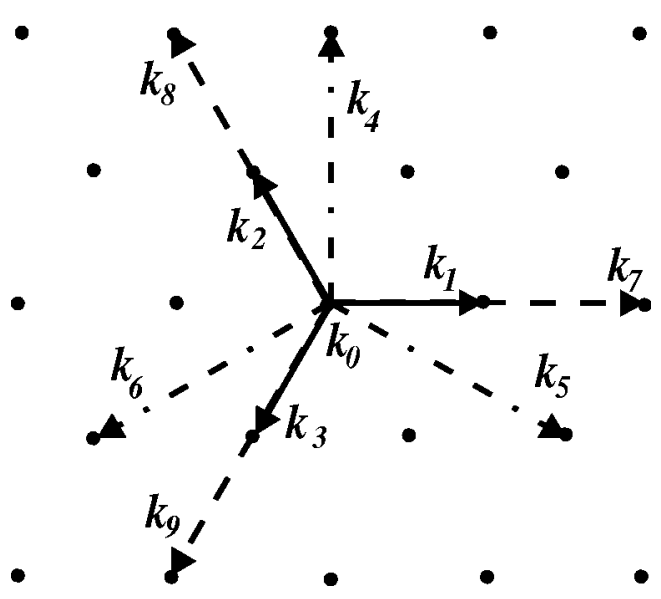

FIG. 1. Stable and unstable modes.

we have taken three vertical eigenmodes, which is enough to ensure a good convergence. Then the bifurcation problem reduces to Eq. (23), with the identification $A_{1} \equiv A_{1}^{k_{1}}, A_{2}$ $\equiv A_{1}^{k_{2}}, A_{3} \equiv A_{1}^{k_{3}}$. (Explicit expressions for nonlinear coefficients in the normal form can be found in Appendix B.) The values of the coefficients in Eq. (23) are given in Table I for pure Marangoni convection $(\mathrm{Ra}=0)$, an insulating interface $(\mathrm{Bi}=0)$, and keeping $g_{1}\left(\operatorname{Pr}^{-1}=0\right)=1$.

\section{B. Spatial terms: The generalized Ginzburg-Landau equation for hexagons}

The stability analysis with respect to inhomogeneous perturbations requires the addition of spatial terms to Eq. (23). Off-critical wave numbers $\mathbf{k}=\mathbf{k}_{c}+\mathbf{q}$ accounting for slight modulations of a perfect pattern change the coefficients in Eq. (23). One can handle these changes by expanding the coefficients in Taylor series of $\mathbf{q}$, performing the calculations and coming back to real space through $\mathbf{q} \rightarrow i \boldsymbol{\nabla}$.

The linear spatial term is easily obtained from the growth rate $\sigma\left(k^{2}, \epsilon\right)=\left[\epsilon-\xi_{0}^{2}\left(k^{2}-k_{c}^{2}\right)^{2} / 4 k_{c}^{2}\right], \xi_{0}^{2}=\partial^{2} \epsilon /\left.\left(2 \partial k^{2}\right)\right|_{k_{c}}$ being the correlation length. To lowest order $\left(k^{2}-k_{c}^{2}\right)^{2}$ $\simeq 4 k_{c}^{2}(\hat{\mathbf{n}} \cdot \mathbf{q})^{2}$, which in real space becomes the usual diffusive linear term $\xi_{0}^{2}(\hat{\mathbf{n}} \cdot \nabla)^{2}$. The values of $\xi_{0}^{2}$ as a function of $\Gamma$ are gathered in Table II . It is worth noticing that $\xi_{0}^{2}$ does not change significantly when $\Gamma$ is varied.

For a pattern of rolls this is the only spatial contribution to the GLE. However, nonlinear gradient terms of the form $A \boldsymbol{\nabla} A$ ought to be included for a subcritical bifurcation, as has been remarked recently by several authors [27-29]. These terms are found after replacing sums by integrals and expanding coefficients $\mathcal{B}$ in Eq. (28) in series of $\mathbf{q}$ and fol-

TABLE I. Coefficients of the amplitude equations for $\mathrm{Ra}=0$ and $\mathrm{Bi}=0$, taking $g_{1}\left(\operatorname{Pr}^{-1}=0\right)=1$.

\begin{tabular}{cc}
\hline \hline$\tau_{0}$ & $\alpha$ \\
\hline $0.167+0.0427 \operatorname{Pr}^{-1}$ & $0.293-0.0666 \operatorname{Pr}^{-1}$ \\
$g_{1}$ & $g_{2}$ \\
$1+0.284 \mathrm{Pr}^{-1}+0.0289 \mathrm{Pr}^{-2}$ & $1.35+0.450 \mathrm{Pr}^{-1}+0.03009 \mathrm{Pr}^{-2}$ \\
\hline \hline
\end{tabular}

TABLE II. Values of $\xi_{0}^{2}$ as a function of $\Gamma$ for $\mathrm{Bi}=0.1$.

\begin{tabular}{ccccccc}
\hline \hline$\Gamma$ & 0 & $\tan \left(10^{\circ}\right)$ & $\tan \left(20^{\circ}\right)$ & $\tan \left(40^{\circ}\right)$ & $\tan \left(70^{\circ}\right)$ & $\tan \left(90^{\circ}\right)$ \\
\hline$\xi_{0}^{2}$ & 0.279 & 0.274 & 0.284 & 0.285 & 0.282 & 0.274 \\
\hline \hline
\end{tabular}

lowing a procedure similar to that for the linear term. (Detailed calculations are given in Appendix B.) Finally, the generalized GLE is obtained

$$
\begin{aligned}
\tau_{0} \partial_{t} A_{1}= & \epsilon A_{1}+\xi_{0}^{2}\left(\hat{\mathbf{n}}_{1} \cdot \boldsymbol{\nabla}\right)^{2} A_{1}+\alpha_{0} \bar{A}_{2} \bar{A}_{3}-g_{1}\left|A_{1}\right|^{2} A_{1} \\
& -g_{2}\left(\left|A_{2}\right|^{2}+\left|A_{3}\right|^{2}\right) A_{1}+i \alpha_{1}\left[\bar{A}_{2}\left(\hat{\mathbf{n}}_{3} \cdot \boldsymbol{\nabla}\right) \bar{A}_{3}\right. \\
& \left.+\bar{A}_{3}\left(\hat{\mathbf{n}}_{2} \cdot \boldsymbol{\nabla}\right) \bar{A}_{2}\right]+i \alpha_{2}\left[\bar{A}_{2}\left(\hat{\boldsymbol{\tau}}_{3} \cdot \boldsymbol{\nabla}\right) \bar{A}_{3}\right. \\
& \left.-\bar{A}_{3}\left(\hat{\boldsymbol{\tau}}_{2} \cdot \boldsymbol{\nabla}\right) \bar{A}_{2}\right],
\end{aligned}
$$

where $\hat{\mathbf{n}}_{i}$ and $\hat{\boldsymbol{\tau}}_{i}$ stand for unit vectors parallel and perpendicular to the wave numbers (see Fig. 2) and $\alpha_{1}$ and $\alpha_{2}$ are real coefficients given by

$$
\alpha_{1}=\left(\frac{\partial \alpha}{\partial k_{2}}+\frac{1}{2} \frac{\partial \alpha}{\partial k_{1}}\right), \quad \alpha_{2}=\frac{\sqrt{3}}{2} \frac{\partial \alpha}{\partial k_{1}},
$$

where $\alpha\left(k_{1}, k_{2}, k_{3}\right)=\tau_{0} \mathcal{B}_{111}\left(k_{1}, k_{2}, k_{3}\right)$ (see Appendix B). The terms with $\alpha_{1}$ and $\alpha_{2}$ render the system nonpotential [27] and correspond to dilatations and distortions of the hexagons, respectively. A sketch of their action in Fourier space is drawn in Fig. 3.

The values of $\alpha_{1}$ and $\alpha_{2}$ as functions of $\Gamma$ are displayed in Fig. 4. As expected, both $\alpha_{0}$ and $\alpha_{1}$ vanish for buoyancydriven convection $(\Gamma \rightarrow 0)$, the bifurcation becoming then supercritical. However, $\alpha_{2}$ remains different from zero [29]. In other ranges of $\Gamma, \alpha_{1}$ and $\alpha_{2}$ are of the same order as $\alpha_{0}$. The values of the coefficients $\alpha_{i}$ do not vary much in the Marangoni-dominated regime, i.e, for $\tan ^{-1}(\Gamma)>45^{\circ}$.

In Table III our values for $\alpha_{1}$ and $\alpha_{2}$ are compared with those found by other authors for $\mathrm{Ra}=0[17,18]$. We have taken the same set of parameters as in Ref. [17], but the method used to derive the amplitude equations is different. We find a disagreement of about a factor of 4 between the two papers, although the sign of the coefficients coincide. In

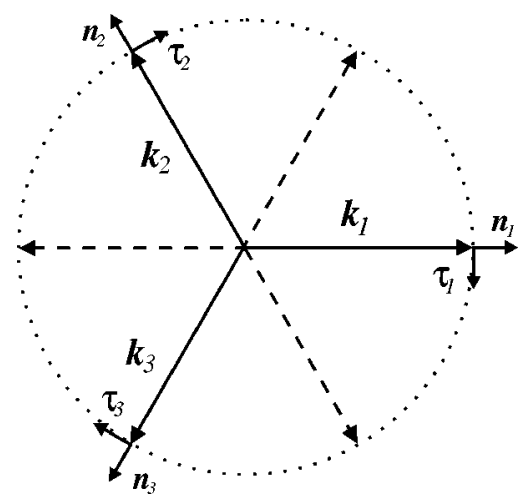

FIG. 2. Unit vectors: $\hat{\mathbf{n}}_{i}$ parallel and $\hat{\boldsymbol{\tau}}_{i}$ perpendicular to the wave numbers of the hexagonal lattice. 

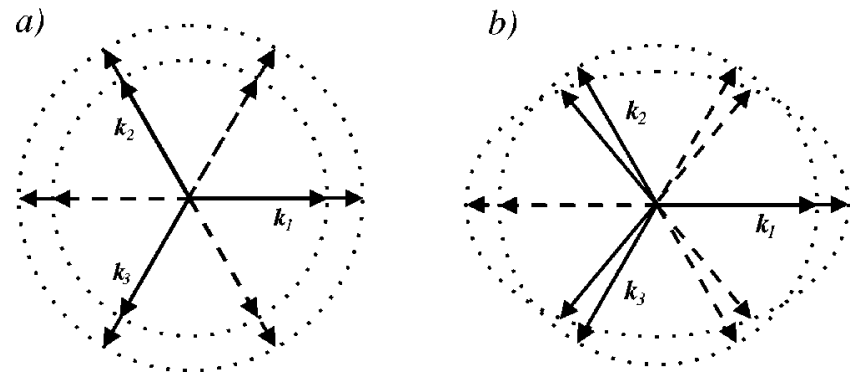

FIG. 3. (a) Dilatations and (b) distortions in a hexagonal pattern.

Ref. [18] the two-fluid problem is analyzed for the conditions in two experiments (Ref. [22] in case (a) and Ref. [30] in case (b)) and then the comparison with our results is less straightforward. Nevertheless, we think that the conditions are rather similar to ours (except perhaps for Bi). The values almost coincide, the main difference being the sign of $\alpha_{1}$. We will discuss this disagreement in the final section.

Once all the coefficients in Eq. (29) have been calculated, we address the stability of the stationary solutions.

\section{AMPLITUDE INSTABILITIES}

The bifurcation diagram of BM convection under homogeneous perturbations is well known. Here we extend it including slightly off-critical wave numbers in the amplitudes $A_{i}=\mathcal{A}_{i} \exp i \mathbf{q}_{i} \cdot \mathbf{x}$, so that solutions of Eq. (29) are easily found. These are (a) rolls $\mathcal{A}_{1}=R, \mathcal{A}_{2}=\mathcal{A}_{3}=0$, with $R$ $=\sqrt{\left(\epsilon-\xi_{0}^{2} q^{2}\right) / g_{1}}$ and hexagons $\mathcal{A}_{1}=\mathcal{A}_{2}=\mathcal{A}_{3}=H$ with $H$ given by

$$
H=\frac{\alpha^{\prime}(q) \pm \sqrt{\alpha^{\prime}(q)^{2}+4\left(\epsilon-\xi_{0}^{2} q^{2}\right)\left(g_{1}+2 g_{2}\right)}}{2\left(g_{1}+2 g_{2}\right)},
$$

in which $\alpha^{\prime}(q)=\alpha_{0}+2 q \alpha_{1}$ and the sign $+(-)$ corresponds to up(down)-hexagons with up(down)-flow motions in the center. The condition $\alpha_{1}>0$ is ensured in BM convection (Fig. 4), so $\alpha^{\prime}(q)$ normally remains positive, and therefore only upflow hexagons $(H>0)$ are stable. Mixed modes of the form $\mathcal{A}_{1}=r_{1}, \mathcal{A}_{2}=\mathcal{A}_{3}=r_{2}$ are also solutions, but they are linearly unstable with respect to hexagons or rolls.

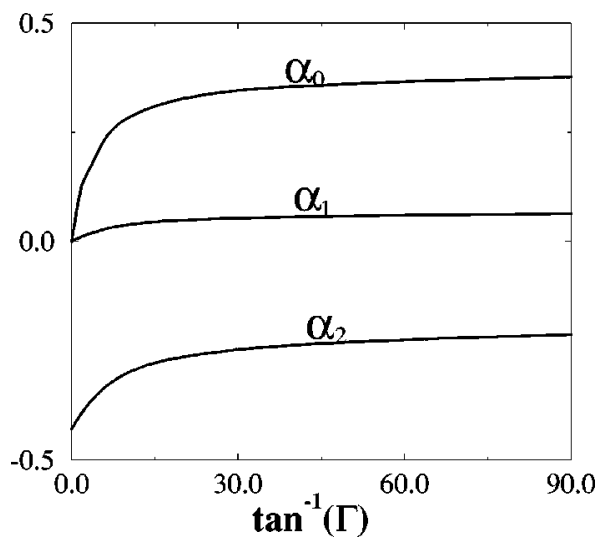

FIG. 4. Coefficients $\alpha_{0}, \alpha_{1}$, and $\alpha_{2}$ as functions of $\Gamma\left(\operatorname{Pr}^{-1}\right.$ $=0, \mathrm{Bi}=0.1$, and $\left.g_{1}=1\right)$.
TABLE III. Values of $\alpha_{1}$ and $\alpha_{2}$ in different papers for pure Marangoni convection $(\mathrm{Ra}=0)$, with the normalization $\xi_{0}^{2}=1, \alpha_{0}$ $=1$. (In the present paper and in Ref. [17] the value $\mathrm{Bi}=0$ is chosen.)

\begin{tabular}{lcccc}
\hline \hline Reference & & $\alpha_{1}$ & $\alpha_{2}$ & Pr \\
\hline Bragard and Velarde $^{\mathrm{a}}$ & & 1.213 & -4.4149 & $\infty$ \\
Golovin et al. $^{\mathrm{b}}$ & (a) & -0.4622 & -1.0832 & 913 \\
& (b) & -0.5572 & -1.3057 & 100 \\
This paper & & 0.3229 & -1.079 & $\infty$ \\
\hline
\end{tabular}

${ }^{\mathrm{a}}$ Reference [17].

${ }^{\mathrm{b}}$ Reference [18].

A linear stability analysis with respect to homogeneous perturbations (amplitude instabilities) in the form (a) $\mathcal{A}_{i}$ $=H\left(1+r_{i}\right)$ for hexagons and (b) $\mathcal{A}_{1}=R\left(1+r_{1}\right), \mathcal{A}_{2,3}=r_{2,3}$ for rolls, is easily performed. Hexagons turn out to be stable if the following conditions

$$
\begin{gathered}
u=H^{2}\left(g_{1}-g_{2}\right)+\alpha^{\prime}(q) H>0, \\
v=2 H^{2}\left(g_{1}+2 g_{2}\right)-\alpha^{\prime}(q) H>0,
\end{gathered}
$$

are satisfied. Similarly the stability of rolls is limited by the curve

$$
m=R^{2}\left(g_{1}-g_{2}\right)+\alpha^{\prime}(q) R<0 .
$$

Hexagons are then stable in the region

$$
\begin{aligned}
\boldsymbol{\epsilon}_{s}(q) & =-\frac{\alpha^{\prime 2}(q)}{4\left(g_{1}+2 g_{2}\right)}+\xi_{0}^{2} q^{2}<\epsilon \\
& <\epsilon_{h}(q) \\
& =\frac{\alpha^{\prime 2}(q)\left(g_{2}+2 g_{1}\right)}{\left(g_{2}-g_{1}\right)^{2}}+\xi_{0}^{2} q^{2},
\end{aligned}
$$

and rolls for

$$
\boldsymbol{\epsilon}>\boldsymbol{\epsilon}_{r}(q)=\frac{\alpha^{\prime 2}(q) g_{1}}{\left(g_{2}-g_{1}\right)^{2}}+\xi_{0}^{2} q^{2} .
$$

(Notice that these expressions do not contain $\alpha_{2}$ since only perfect equilateral hexagons have been considered.) A sketch of the bifurcation diagram for fixed $q$ is shown in Fig. 5 .

In Fig. 6 we represent $\epsilon_{s}, \epsilon_{r}$, and $\epsilon_{h}$ as functions of $\Gamma$ for $q=0$. These curves do not vary much for $\tan ^{-1} \Gamma \geqslant 30^{\circ}$ (i.e., in the Marangoni-dominated regime), but they steeply descend for $\tan ^{-1} \Gamma<30^{\circ}$. In the limit $\tan ^{-1} \Gamma \rightarrow 0$ they vanish, rolls then becoming the only stable pattern as predicted for RB convection. A similar behavior was obtained for coefficients $\alpha_{i}$ (see Fig. 4). The curves in Fig. 6 do not display a sudden threshold from a Rayleigh to a Marangoni-dominated regime, since the main changes are produced between the two limits discussed above.

The variation of $\epsilon_{s}, \epsilon_{r}$, and $\epsilon_{h}$ with $\operatorname{Pr}$ for $\mathrm{Ra}=0$ and $\mathrm{Bi}=0$ is gathered in Fig. 7. We have taken the eigenfunctions for $\operatorname{Pr}^{-1}=0$, so our results are not expected to be valid 


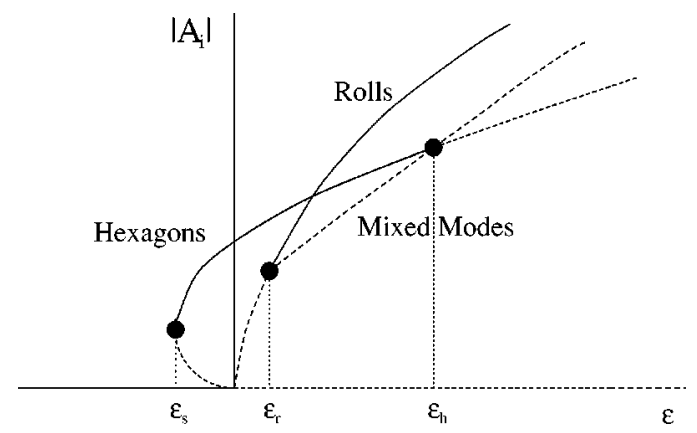

FIG. 5. Bifurcation diagram. Solid lines indicate stable solutions and dotted ones unstable.

for $\operatorname{Pr} \ll 1$. However, even for rather small Pr they are similar to those obtained by several authors using other methods $[31,32]$. In particular they noticed that the quadratic coefficient $\alpha$ changes sign, inducing a transition from up to down hexagons for $\operatorname{Pr}_{c} \sim 0.23$ [31,32], in complete agreement with the value $\operatorname{Pr}_{c}=0.227$ in our calculation. Notice that threshold values in Fig. 7 do not vary appreciably for $\operatorname{Pr}>5$, so that we do not expect qualitative changes for sufficiently high Pr. Nevertheless we must mention that mean-flow effects become more important as Pr is decreased. These enter into the description through a pair of coupled amplitude equations the derivation of which is rather involved [1].

A comparison with previous works $[13,15,17]$ for the set of parameters $\mathrm{Ra}=0, \mathrm{Pr}^{-1}=0, \mathrm{Bi}=0$, and $q=0$ is gathered in Table IV. Obviously the best agreement is with Ref. [15] because we used the same method to obtain the coefficients (although we take slightly different eigenfunctions).

Hexagons have been observed to be replaced by squares in recent BM experiments $[37,38]$. Numerical simulations [39] confirmed also that this transition is possible for $\epsilon \approx 3$ in liquids with $\operatorname{Pr} \approx 100$, in rather good agreement with experimental data. Other theoretical studies [40] have shown such a transition by increasing $\mathrm{Bi}$ but beyond the value estimated from experiments. In principle the perturbative calculations in the present paper could be extended to include destabilization of hexagons by squares. But an agreement with experiments is not hoped to be achieved unless finite-Pr effects
TABLE IV. Comparison among the values of $\epsilon_{s}, \epsilon_{r}$, and $\epsilon_{h}$ for pure Marangoni convection $(\Gamma=\infty)$, with $\operatorname{Pr}^{-1}=0$ and $\mathrm{Bi}=0$.

\begin{tabular}{lccc}
\hline \hline Reference & $\epsilon_{s}(\%)$ & $\epsilon_{r}$ & $\epsilon_{h}$ \\
\hline Bragard and Lebon $^{\mathrm{a}}$ & -0.56 & 0.53 & 1.8 \\
Thess and Orszag $^{\mathrm{b}}$ & -0.75 & & \\
${\text { Parmetier } \text { et al. }^{\mathrm{c}}}$ & -0.58 & 0.71 & 2.37 \\
Bragard and Velarde $^{\mathrm{d}}$ & -0.57 & 0.64 & 2.16 \\
This paper & -0.58 & 0.70 & 2.34 \\
\hline \hline
\end{tabular}

${ }^{\mathrm{a}}$ Reference [13].

${ }^{\mathrm{b}}$ Reference [14].

${ }^{\mathrm{c}}$ Reference [15].

${ }^{\mathrm{d}}$ Reference [17].

and a full two-layer system would be considered. Obviously these two facts will render calculations much more cumbersome. For example, the second effect has been taken into account by means of the amplitude equations for two-layer BM convection in a recent article [41]. In this case, hexagons are replaced by squares but for $\epsilon>1$, i.e, outside the validity of the perturbation approach [41].

\section{PHASE INSTABILITIES}

Another kind of destabilizing perturbation is in the form to long-wave modulations whose dynamics is governed by a phase equation. The relevance of this equation for rolls was soon noticed [26], but it took somewhat longer to determine it for hexagons [33,19-21]. In the framework of the amplitude equations (but for $\alpha_{1}=\alpha_{2}=0$ ) the phase equation for hexagons was obtained in [19-21]. Other authors have studied the influence of the quadratic spatial terms on the dispersion relation associated with Eq. (29) $\left(\alpha_{1}, \alpha_{2} \neq 0\right)[17,34]$. For this general case an explicit derivation of the phase equation with coefficients computed analytically can be found in Ref. [35].

Assuming perturbations of Eq. (29) in the form $\mathcal{A}_{i}$ $=H\left(1+r_{i}+i \phi_{i}\right)$, with $r_{i}$ the amplitude and $\phi_{i}$ the phase of the perturbation and linearizing, we arrive at the system
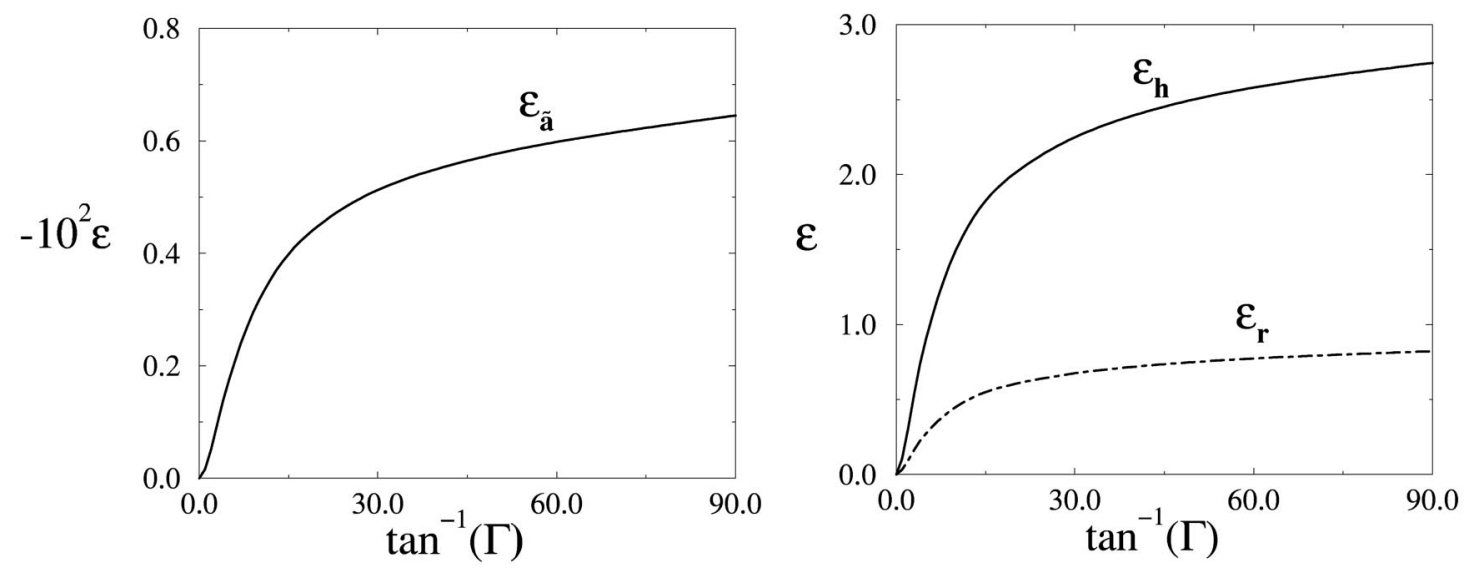

FIG. 6. Values of $\epsilon_{s}, \epsilon_{r}$, and $\epsilon_{h}$ as functions of $\tan ^{-1}(\Gamma)\left(\operatorname{Pr}^{-1}=0\right.$ and $\left.\mathrm{Bi}=0.1\right)$. 

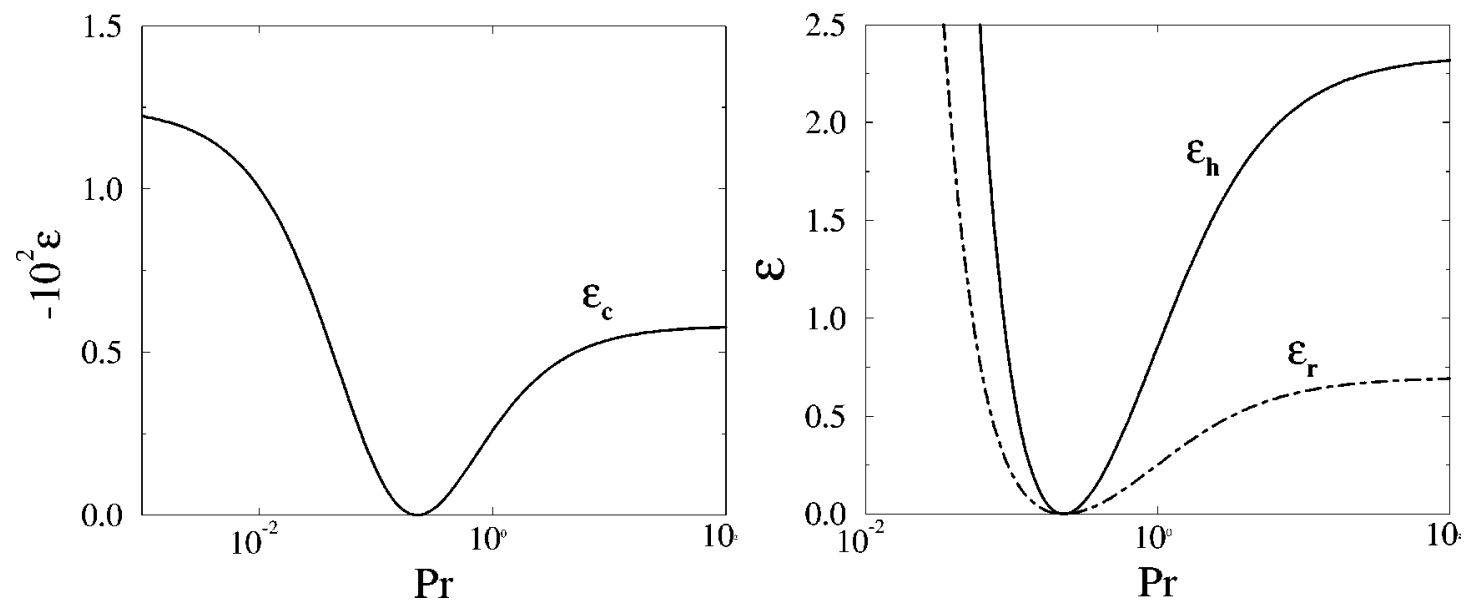

FIG. 7. Dependence of $\epsilon_{s}, \epsilon_{r}$, and $\epsilon_{h}$ on $\operatorname{Pr}$ for $\mathrm{Ra}=0$ and $\mathrm{Bi}=0$.

$$
\begin{aligned}
\tau_{0} \partial_{t} r_{1}= & \xi_{0}^{2} \partial_{1}^{2} r_{1}-2 q \xi_{0}^{2} \partial_{1} \phi_{1}+\alpha^{\prime}(q) H\left(r_{2}+r_{3}-r_{1}\right)+H \\
& \times\left(\alpha_{1}+\frac{\alpha_{2}}{\sqrt{3}}\right)\left(\partial_{2} \phi_{2}+\partial_{3} \phi_{3}\right)+\alpha_{2} H\left(\partial_{3} \phi_{2}+\partial_{3} \phi_{3}\right) \\
- & 2 g_{1} H^{2} r_{1}-2 g_{2} H^{2}\left(r_{2}+r_{3}\right) \\
\tau_{0} \partial_{t} \phi_{1}= & 2 q \xi_{0}^{2} \partial_{1} r_{1}+\xi_{0}^{2} \partial_{1}^{2} \phi_{1}-\alpha^{\prime}(q) H\left(\phi_{1}+\phi_{2}+\phi_{3}\right) \\
& +H\left(\alpha_{1}+\frac{\alpha_{2}}{\sqrt{3}}\right)\left(\partial_{2} r_{2}+\partial_{3} r_{3}\right)+\frac{2}{\sqrt{3}} \alpha_{2} H \\
& \times\left(\partial_{2} r_{3}+\partial_{3} r_{2}\right)
\end{aligned}
$$

where the notation $\partial_{i}=\hat{\mathbf{n}}_{i} \cdot \boldsymbol{\nabla}$ is used. The amplitudes $r_{i}$ and the global phase $\Phi=\phi_{1}+\phi_{2}+\phi_{3}$ are strongly damped modes and can be eliminated adiabatically. As a result, the dynamics are dominated by two phase modes. Instead of using $\phi_{2}$ and $\phi_{3}$ we deal with $\phi_{x}=-\left(\phi_{2}+\phi_{3}\right), \phi_{y}$ $=1 / \sqrt{3}\left(\phi_{2}-\phi_{3}\right)$, that are related to the two translational symmetries in the $x$ and $y$ directions, respectively. The final equation reads as

$$
\partial_{t} \vec{\phi}=D_{t} \nabla^{2} \vec{\phi}+\left(D_{l}-D_{t}\right) \boldsymbol{\nabla}(\boldsymbol{\nabla} \cdot \vec{\phi}),
$$

where the diffusion coefficients $D_{t}$ and $D_{l}$ are analogous to the velocity of transversal and longitudinal sound waves in an isotropic solid, respectively.

This equation can be formally obtained just by symmetry arguments and therefore it is valid even far from onset. Moreover, it can be extended to include nonlinear terms [21]. But the coefficients are difficult to calculate unless the amplitude equation is used. In this framework Eq. (29) leads to the analytical expressions [35]:

$$
D_{t}=\frac{1}{4}-\frac{q^{2}}{2 u}+\frac{H^{2}}{8 u}\left(\alpha_{1}-\sqrt{3} \alpha_{2}\right)^{2}
$$

$$
\begin{aligned}
D_{l}= & \frac{3}{4}-\frac{q^{2}(4 u+v)}{2 u v}+\frac{H^{2}}{8 u}\left(\alpha_{1}-\sqrt{3} \alpha_{2}\right)^{2}-\frac{H^{2} \alpha_{1}}{v}\left(\alpha_{1}\right. \\
& \left.+\sqrt{3} \alpha_{2}\right)+\frac{H q}{v}\left(3 \alpha_{1}+\sqrt{3} \alpha_{2}\right),
\end{aligned}
$$

where $u=0$ and $v=0$ are defined in Eqs. (32),(33).

Using the analogy with sound waves we split the phase $\vec{\phi}$ into a longitudinal $\vec{\phi}_{l}$ and a transversal $\vec{\phi}_{t}$ part, satisfying $\boldsymbol{\nabla} \times \vec{\phi}_{l}=0$ (rhombic distortions) and $\boldsymbol{\nabla} \cdot \vec{\phi}_{t}=0$ (rectangular distortions). These components satisfy

$$
\partial_{t} \vec{\phi}_{l}=D_{l} \nabla^{2} \vec{\phi}_{l}, \quad \partial_{t} \vec{\phi}_{t}=D_{t} \nabla^{2} \vec{\phi}_{t} .
$$

Therefore the system is stable to phase perturbations provided that $D_{t}>0$ and $D_{l}>0$. It should be noted, however, that these conditions do not necessarily give the right stability limits. (In fact, it has been shown that oscillatory, as well as shortwave instabilities can appear under some special circumstances [36].) In order to ensure that the correct stability limits are obtained, we solve also the full $6 \times 6$ dispersion relation corresponding to Eq. (29). In Fig. 8 we show the phase stability diagrams for several values of $\Gamma$, as well as the results from the dispersion relation (circles). For the sake of comparison the amplitude stability curves $u=0$ (lower dotted curve) and $v=0$ (higher dotted curve), together with the roll amplitude limit $m=0$ (dashed-dotted middle line) have been included. Figure 8(a), which corresponds to buoyancy-dominated convection, shows a small, almost symmetric stability region. This becomes big and asymmetric in Figs. 8(b)-8(d), in the Marangoni-dominated regime. This behavior is in agreement with the variations in $\epsilon_{i}$ and $\alpha_{i}$ seen in Figs. 4 and 6. Those stability regions are bent to the right, mainly owing to the positive sign of $\alpha_{1}$. A similar bend is observed in numerical calculations [16,17], a fact related apparently to a wave number growth when Ma increases [5]. 

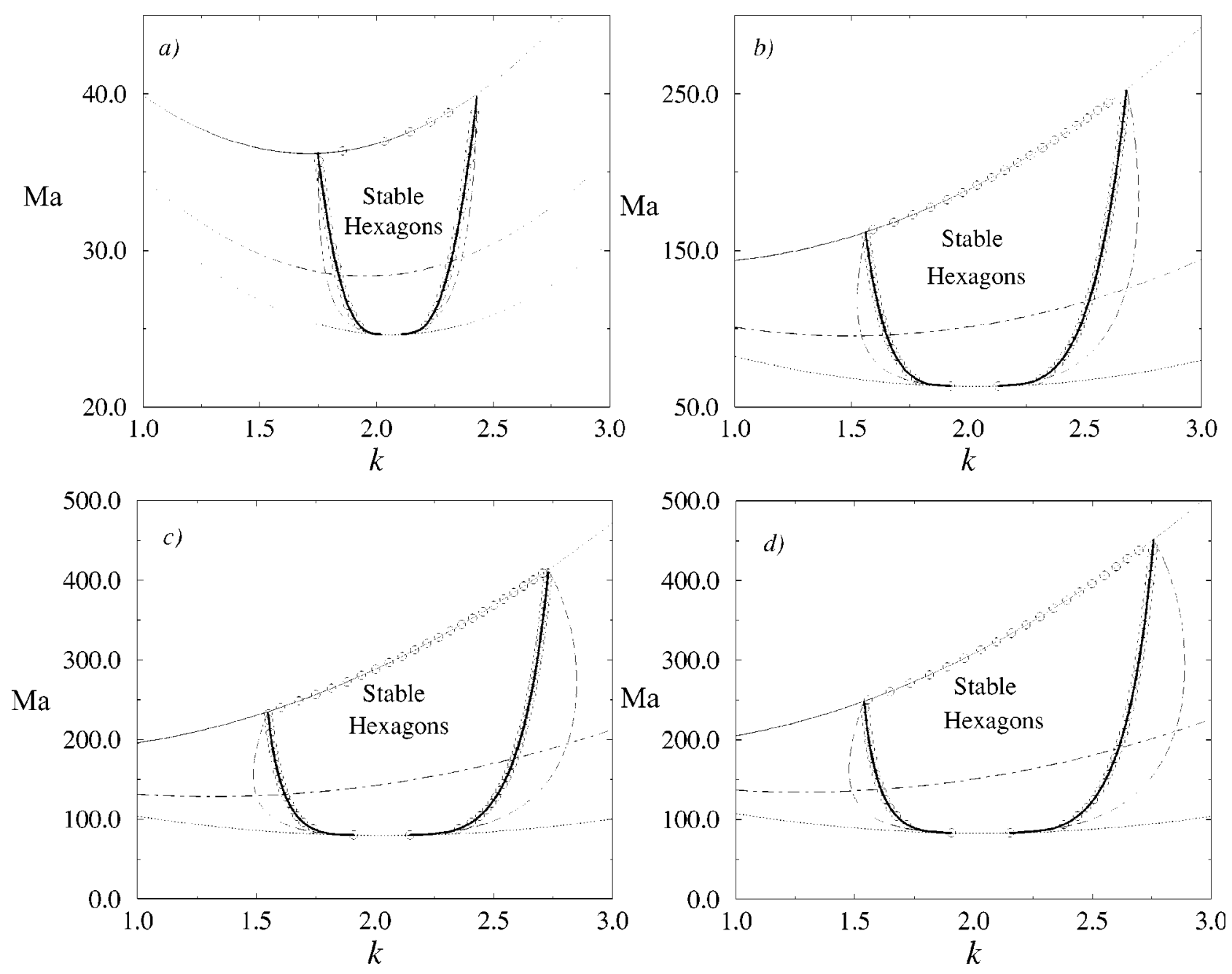

FIG. 8. Phase stability diagram for (a) $\Gamma=0.05$, (b) $\Gamma=\tan \left(20^{\circ}\right)$, (c) $\Gamma=\tan \left(70^{\circ}\right)$, and (d) $\Gamma=\tan \left(90^{\circ}\right)\left(\operatorname{Pr}^{-1}=0, B i=0.1\right)$.

\section{CONCLUSIONS}

In this paper we have studied the instabilities of hexagonal patterns in BM convection, in the weakly nonlinear regime. We have obtained generalized Ginzburg-Landau equations governing the slow dynamics of hexagon amplitudes near onset, which include spatial nonlinear terms. We have computed the coefficients of the nonlinear gradient terms, showing that the three coefficients $\alpha_{i}$ are of the same order, their value remaining almost constant (like the threshold $\epsilon_{i}$ ) when the liquid depth is varied in the Marangoni-dominated regime.

The stability analysis of the spatially homogeneous case is in good agreement with previous theoretical results $[12,13,15]$. From Fig. 6 we can conclude that the threshold values $\epsilon_{s}, \epsilon_{r}, \epsilon_{h}$ do not depend so much on the liquid depth in the Marangoni regime, their changes becoming significant for thick layers (buoyancy-dominated regime). A comparison with experiments [7] is not yet easy. With the data obtained so far only $\epsilon_{c}$ could be compared, but its tiny value might be influenced by finite-size effects. (A experimental setup measuring the Nusselt number would permit a full quantitative comparison.)

The new spatial terms modify slightly the amplitude sta- bility curves (see Fig. 8). These curves describe a transition between hexagons and rolls. Experimentally, however, a change of hexagons into squares has been reported by two groups $[37,38]$ for values of $\epsilon=\mathrm{Ma}-\mathrm{Ma}_{c} / \mathrm{Ma}_{c} \geqslant 3$. A similar value is obtained by direct numerical simulations of hydrodynamic equations [39]. Although our analysis could be extended to account for a transition to squares, the corresponding values would lie outside the validity of a perturbative approach.

But the main contribution of the coefficients $\alpha_{i}$ becomes apparent when dealing with long-wave perturbations. We derive a phase equation for hexagons and calculate its coefficients in an analytical form. This equation allows us to determine phase-stable regions that fit qualitatively with those computed numerically by other authors $[16,17]$.

In particular we have shown that the phase-stability regions do not change qualitatively by increasing $\Gamma$ within the Marangoni-dominated regime, in agreement with numerical calculations in Ref. [16]. This suggests that the long-wave dynamics of hexagonal patterns can be studied even in relatively thick liquid layers ( $d \leqslant 8 \mathrm{~mm}$ for typical fluids).

Finally, let us mention that the perturbations considered in this paper seem to be the most dangerous for a hexagonal 
pattern, described by amplitude equations [28]. Although the stability diagrams derived with this formalism have a limited range of validity we hope that they will suggest further experiments on thresholds, transitions, and sideband instabilities of hexagon patterns in BM convection.

\section{ACKNOWLEDGMENTS}

We thank H. Mancini and B. Peña (Pamplona), J. Bragard (Boston), G. Lebon (Liège), and M. Bestehorn (Sttutgart) for many fruitful discussions and comments. This work was supported by the DGICYT (Spanish Government) under Grant No. PB98-0208, by PIUNA (Universidad de Navarra). B.E. acknowledges financial support (BFI95.035) from the Basque Government.

\section{APPENDIX A: LINEAR BM INSTABILITY}

\section{Linear adjoint operator}

In this Appendix we complete the calculations sketched in Sec. II A. Let us recall that the linear problem can be written as

$$
\left(\begin{array}{ccc}
\boldsymbol{\nabla}^{2} & \operatorname{Ra}_{z} & -\boldsymbol{\nabla} \\
\mathbf{e}_{z} & \boldsymbol{\nabla}^{2} & 0 \\
\boldsymbol{\nabla} & 0 & 0
\end{array}\right)\left(\begin{array}{c}
\mathbf{v} \\
\theta \\
\pi
\end{array}\right)=\sigma\left(\begin{array}{ccc}
\operatorname{Pr}^{-1} & 0 & 0 \\
0 & 1 & 0 \\
0 & 0 & 0
\end{array}\right)\left(\begin{array}{c}
\mathbf{v} \\
\theta \\
\pi
\end{array}\right) .
$$

Using the scalar product in Eq. (2.1) and the condition for the adjoint operator $\left\langle f^{*}, \mathcal{L}_{c} f\right\rangle=\left\langle f, \mathcal{L}_{c}^{*} f^{*}\right\rangle$ we obtain

$$
\mathcal{L}^{*}=\left(\begin{array}{ccc}
\boldsymbol{\nabla}^{2} & \mathbf{e}_{z} & -\boldsymbol{\nabla} \\
\operatorname{Ra}_{c} \mathbf{e}_{z} & \boldsymbol{\nabla}^{2} & 0 \\
\boldsymbol{\nabla} & 0 & 0
\end{array}\right)
$$

with the bc

$$
\begin{gathered}
u^{*}=v^{*}=w^{*}=\theta^{*}=0, \text { at } z=0, \\
\partial_{z} \theta^{*}+\operatorname{Bi} \theta^{*}+\operatorname{Ma}_{c}\left(\partial_{x} u^{*}+\partial_{y} v^{*}\right)=0, \\
\partial_{z} u^{*}=\partial_{z} v^{*}=w^{*}=0, \text { at } z=1,
\end{gathered}
$$

where $f^{*}=\left(\mathbf{v}^{*}, \pi^{*}, \theta^{*}\right)$ are the adjoint eigenfunctions. Expanding the fields into normal modes $\left(\mathbf{v}^{*}, \theta^{*}, \pi^{*}\right)$ $=\left[k^{2} \mathbf{V}_{\gamma}^{k *}(z), \Theta_{\gamma}^{k *}(z), \Pi_{\gamma}^{k *}(z)\right] e^{i \mathbf{k} \cdot \mathbf{x}}$ the former system becomes

$$
\begin{gathered}
\left(D^{2}-k^{2}\right)^{2} W_{\gamma}^{k *}-\Theta_{\gamma}^{k *}=\operatorname{Pr}^{-1} \sigma_{\gamma}^{k *}\left(D^{2}-k^{2}\right) W_{\gamma}^{k *}, \\
\left(D^{2}-k^{2}\right) \Theta_{\gamma}^{k *}+\operatorname{Ra}_{c} k^{2} W_{\gamma}^{k *}=\sigma_{\gamma}^{k *} \Theta_{\gamma}^{k *},
\end{gathered}
$$

with the bc

$$
\begin{gathered}
\Theta_{\gamma}^{k *}(0)=D \Theta_{\gamma}^{k *}(1)+\mathrm{Bi}_{\gamma}^{k *}(1)-\mathrm{Ma}_{c} k^{2} D W_{\gamma}^{k *}(1)=0 \\
W_{\gamma}^{k *}(0)=D W_{\gamma}^{k *}(0)=W_{\gamma}^{k *}(1)=D^{2} W_{\gamma}^{k *}(1)=0
\end{gathered}
$$

The corresponding eigenvalue problem satisfies the relation $\bar{\sigma}_{\gamma}^{k *}=\sigma_{\gamma}^{k}$, from which it is easy to derive the orthogonality condition:

$$
\left\langle\operatorname{Pr}^{-1} \mathbf{v}_{\gamma}^{k_{i}^{*}} \cdot \mathbf{v}_{\rho}^{k_{j}}+\theta_{\gamma}^{k_{i}^{*}} \theta_{\rho}^{k_{j}}\right\rangle=0 \text { if } \gamma \neq \rho \text { or } k_{i} \neq k_{j} .
$$

The adjoint eigenfunctions are solutions of the system (A5)(A8). They will be used in computing the coefficients in the amplitude equations.

\section{Eigenmode $\boldsymbol{k}=\mathbf{0}$}

As discussed in Ref. [15], the limit $k \rightarrow 0$ in Eqs. (17) and (18) is singular, so it must be computed separately. Setting $k=0$, Eqs. (17) and (18) become

$$
\begin{aligned}
& D^{4} W=\sigma D^{2} W \\
& \left(\sigma-D^{2}\right) \Theta=W
\end{aligned}
$$

with the bc (19) and (20) for $k=0$. The solution of this system is

$$
W_{\gamma}(z) \equiv 0, \quad \Theta_{\gamma}(z)=\sin \sqrt{-\sigma_{\gamma}} z
$$

where $\sigma_{\gamma}$ satisfies $\sqrt{-\sigma_{\gamma}} \cos \sqrt{-\sigma_{\gamma}}=-\mathrm{Bi} \sin \sqrt{-\sigma_{\gamma}}$. For $\mathrm{Bi}=0, \sigma_{\gamma}=-(\gamma+1 / 2)^{2} \pi^{2}$.

\section{Linear coefficients in the normal form}

The linear analysis is completed by giving an explicit expression for the relaxation time $\tau_{0}$ and the correlation length $\xi_{0}^{2}$. These are found by expanding the growth rate $\sigma$ in terms of $k^{2}$ and $\epsilon \equiv\left(\mathrm{Ma}-\mathrm{Ma}_{c}\right) / \mathrm{Ma}_{c}=\left(\mathrm{Ra}-\mathrm{Ra}_{c}\right) / \mathrm{Ra}_{c}$. After using $\sigma\left(k_{c}, \epsilon=0\right)=\partial \sigma /\left.\partial k^{2}\right|_{k=k_{c}}=0$, and

$$
\left.\frac{\partial^{2} \sigma}{\partial\left(k^{2}\right)^{2}}\right|_{k=k_{c}}=-\left.\left.\frac{\partial \sigma}{\partial \epsilon}\right|_{\epsilon=0} \frac{\partial^{2} \epsilon}{\partial\left(k^{2}\right)^{2}}\right|_{\sigma=0}
$$

one arrives at the expression

$$
\begin{aligned}
\sigma\left(k^{2}, \boldsymbol{\epsilon}\right) & =\left.\frac{\partial \sigma}{\partial \boldsymbol{\epsilon}}\right|_{\boldsymbol{\epsilon}=0}\left[\boldsymbol{\epsilon}-\left.\frac{\partial^{2} \boldsymbol{\epsilon}}{\partial\left(k^{2}\right)^{2}}\right|_{\sigma=0}\left(k^{2}-k_{c}^{2}\right)^{2}\right] \\
& =\tau_{0}^{-1}\left[\boldsymbol{\epsilon}-\frac{\xi_{0}^{2}}{4 k_{c}^{2}}\left(k^{2}-k_{c}^{2}\right)^{2}\right],
\end{aligned}
$$

with $\tau_{0}$ and $\xi_{0}^{2}$ given by

$$
\left.\tau_{0}^{-1} \equiv \frac{\partial \sigma}{\partial \epsilon}\right|_{\epsilon=0},\left.\quad \xi_{0}^{2} \equiv \frac{1}{2} \frac{\partial^{2} \epsilon}{\partial k^{2}}\right|_{k=k_{c}} .
$$

Taking into account that $k=\left|\mathbf{k}_{c}+\mathbf{q}\right|$, then $\left(k^{2}-k_{c}^{2}\right)^{2}$ $=4 k_{c}^{2}\left(\hat{\mathbf{n}} \cdot \mathbf{q}+q^{2} / 2 k_{c}\right)^{2}$ and transforming back to real space this term becomes

$$
\xi_{0}^{2}\left(\hat{\mathbf{n}} \cdot \boldsymbol{\nabla}-\frac{i \boldsymbol{\nabla}^{2}}{2 k_{c}}\right)^{2}
$$


which is the rotationally invariant linear spatial term introduced by Gunaratne et al [28]. In the case of hexagons the term with the Laplacian can be neglected due to the resonant interaction [18].

After projection of Eq. (A1) over the adjoint modes and integrating by parts $\left\langle\theta^{*} \nabla^{2} \theta\right\rangle$ we obtain for $\sigma$

$$
\sigma=\frac{\left\langle\theta^{*} w\right\rangle+\left\langle\nabla^{2} \theta^{*} \theta\right\rangle-\operatorname{Ma}\left\langle\partial_{z} w^{*} \theta\right\rangle_{z=1}-\left\langle\left(\mathbf{v}^{*} \cdot \nabla\right) \pi\right\rangle+\operatorname{Ra}\left\langle w^{*} \theta\right\rangle+\left\langle\mathbf{v}^{*} \cdot \nabla^{2} \mathbf{v}\right\rangle}{\left\langle|\theta|^{2}\right\rangle+\operatorname{Pr}^{-1}\left\langle|\mathbf{v}|^{2}\right\rangle} .
$$

Close to onset $\mathrm{Ma}=\operatorname{Ma}_{c}(1+\epsilon), \operatorname{Ra}=\operatorname{Ra}_{c}(1+\epsilon)$ so, finally we arrive at a useful, explicit expression for $\tau_{0}$

$$
\tau_{0}^{-1}=\frac{\operatorname{Ra}_{c} k_{c}^{2} \int_{0}^{1} W^{*} \Theta d z-\mathrm{Ma}_{c} k_{c}^{2} D W^{*}(1) \Theta(1)}{\int_{0}^{1}\left[|\Theta|^{2}+\operatorname{Pr}^{-1}\left(|D W|^{2}+k_{c}^{2}|W|^{2}\right)\right]} .
$$

\section{APPENDIX B: NONLINEAR TERMS IN THE AMPLITUDE EQUATIONS}

Considering the fields $\psi=[\mathbf{v}, \theta, \pi]$ we can sketch Eqs. (9) in a compact form

$$
\partial_{t} \psi=\mathcal{L}(R, \boldsymbol{\nabla}) \psi+\mathcal{N}(\psi),
$$

where $\mathcal{N}(\psi)$ is a quadratic nonlinearity. Close to onset we consider horizontal slow spatial variations through the expansion $\psi(\mathbf{x}, z, t)=\Sigma_{\gamma, k_{i}} A_{\gamma}^{k_{i}}(\mathbf{x}, t) \mathbf{f}_{\gamma}^{k_{c}}(z) e^{i \mathbf{k}_{c} \cdot \mathbf{x}}$. Moreover, in Fourier space, the planform is composed of finite regions around perfect peaks $\left(\mathbf{k}=\mathbf{k}_{\mathbf{c}}+\mathbf{q}\right)$, so that we can take

$$
A_{\gamma}^{k_{i}}(\mathbf{x}, t)=\int_{q \simeq 0} a_{\gamma}^{q_{i}}(t) e^{i \mathbf{q}_{\mathbf{i}} \cdot \mathbf{x}} d \mathbf{q}
$$

and therefore we can write for $\psi(\mathbf{x}, z, t)$

$$
\begin{aligned}
\psi(\mathbf{x}, z, t) & =\sum_{\gamma} \int a_{\gamma}^{k}(t) \psi_{\gamma}^{k}(\mathbf{x}, z) d \mathbf{k} \\
& =\sum_{\gamma} \int a_{\gamma}^{k}(t) f_{\gamma}^{k}(z) e^{i \mathbf{k} \cdot \mathbf{x}} d \mathbf{k} .
\end{aligned}
$$

Replacing these expansions into Eq. (B1) and projecting over the adjoint modes we arrive at

$$
\begin{aligned}
\dot{a}_{\gamma}^{k}(t)= & \sigma_{\gamma}^{k}(k, R) a_{\gamma}^{k}(t) \\
& +\sum_{\delta, \rho} \int d \mathbf{k}^{\prime} \int d \mathbf{k}^{\prime \prime} \mathcal{B}_{\gamma, \delta, \rho}\left(\mathbf{k}, \mathbf{k}^{\prime}, \mathbf{k}^{\prime \prime}\right) \delta\left(\mathbf{k}-\mathbf{k}^{\prime}\right. \\
& \left.-\mathbf{k}^{\prime \prime}\right) a_{\delta}^{k^{\prime}}(t) a_{\rho}^{k^{\prime \prime}}(t),
\end{aligned}
$$

in which we have defined the coefficient $\mathcal{B}$ as

$$
\mathcal{B}_{\gamma, \delta, \rho}\left(\mathbf{k}, \mathbf{k}^{\prime}, \mathbf{k}^{\prime \prime}\right)=\frac{\left\langle f_{\gamma}^{k *}(z), \mathcal{N}\left[f_{\delta}^{k^{\prime}}(z), f_{\rho}^{k^{\prime \prime}}(z)\right]\right\rangle}{\left\langle f_{\gamma}^{k *}(z), f_{\gamma}^{k}(z)\right\rangle} .
$$

We consider the modes in Fig. 1, which can be classified as unstable $\quad\left[\sigma_{u} \simeq 0,\left(k_{1}, k_{2}, k_{3}\right)\right] \quad$ and stable $\quad\left[\sigma_{s}\right.$ $\left.<0,\left(k_{0}, k_{4}, \ldots, k_{9}\right)\right]$. The latter can be eliminated adiabatically $\left(\dot{a}_{\delta}^{k_{s}} \simeq 0\right)$ :

$$
a_{\delta}^{k_{s}}(t)=-\sum_{\beta, \rho} \int d \mathbf{k}^{\prime \prime} d \mathbf{k}^{\prime \prime \prime} \mathcal{C}_{\delta \beta \rho} \delta\left(\mathbf{k}_{s}-\mathbf{k}^{\prime \prime}-\mathbf{k}^{\prime \prime \prime}\right) a_{\beta}^{k^{\prime \prime}} a_{\rho}^{k^{\prime \prime \prime}},
$$

where $\mathcal{C}_{\delta \beta \rho}\left(\mathbf{k}, \mathbf{k}^{\prime}, \mathbf{k}^{\prime \prime}\right)=\mathcal{B}_{\delta \beta \rho}\left(\mathbf{k}, \mathbf{k}^{\prime}, \mathbf{k}^{\prime \prime}\right) / \sigma_{\delta}^{k}$. This allows to write

$$
\begin{aligned}
\dot{a}_{\gamma}^{k}(t)= & \sigma_{\gamma}^{k}(k, R) a_{\gamma}^{k}(t)+\sum_{\delta \rho} \int d \mathbf{k}^{\prime} \int d \mathbf{k}^{\prime \prime} \mathcal{B}_{\gamma \delta \rho}\left(\mathbf{k}, \mathbf{k}^{\prime}, \mathbf{k}^{\prime \prime}\right) \delta(\mathbf{k} \\
& \left.-\mathbf{k}^{\prime}-\mathbf{k}^{\prime \prime}\right) a_{\delta}^{k^{\prime}}(t) a_{\rho}^{k^{\prime \prime}}(t) \\
& -\sum_{\delta \rho \beta} \int d \mathbf{k}^{\prime} \int d \mathbf{k}^{\prime \prime} \int d \mathbf{k}^{\prime \prime \prime} \delta\left(\mathbf{k}-\mathbf{k}^{\prime}\right. \\
& \left.-\mathbf{k}_{s}\right) \mathcal{D}_{\gamma \delta \rho \beta}\left(\mathbf{k}, \mathbf{k}^{\prime}, \mathbf{k}^{\prime \prime}, \mathbf{k}^{\prime \prime \prime}\right) a_{\delta}^{k^{\prime}}(t) a_{\rho}^{k^{\prime \prime}}(t) a_{\beta}^{k^{\prime \prime \prime}}(t),
\end{aligned}
$$

with

$$
\begin{aligned}
\mathcal{D}_{\gamma \delta \rho \beta}\left(\mathbf{k}, \mathbf{k}^{\prime}, \mathbf{k}^{\prime \prime}, \mathbf{k}^{\prime \prime \prime}\right)= & \sum_{\nu} \int d \mathbf{k}_{s} \delta\left(\mathbf{k}_{s}-\mathbf{k}^{\prime \prime}-\mathbf{k}^{\prime \prime \prime}\right) \\
& \times \mathcal{B}_{\gamma \nu \beta}\left(\mathbf{k}, \mathbf{k}^{\prime}, \mathbf{k}^{\prime \prime}\right) \mathcal{C}_{\nu \delta \rho}\left(\mathbf{k}_{s}, \mathbf{k}^{\prime \prime}, \mathbf{k}^{\prime \prime \prime}\right) .
\end{aligned}
$$

The coefficients $\mathcal{B}$ can be split into a thermal part $\mathcal{B}^{\theta}$ and a viscous part $\mathcal{B}^{v}, \mathcal{B}=\mathcal{B}^{\theta}+\mathcal{B}^{v}$. From Eqs. (8)-(11) one obtains

$$
\begin{gathered}
\mathcal{B}_{\gamma \delta \rho}^{\theta}\left(\mathbf{k}, \mathbf{k}^{\prime}, \mathbf{k}^{\prime \prime}\right) \equiv \frac{\left\langle\theta_{\gamma}^{k *}\left(\mathbf{v}_{\delta}^{k^{\prime}} \cdot \boldsymbol{\nabla}\right) \theta_{\rho}^{k^{\prime \prime}}\right\rangle}{\left\langle\operatorname{Pr}^{-1} \mathbf{v}_{\gamma}^{k *} \cdot \mathbf{v}_{\gamma}^{k}+\theta_{\gamma}^{k *} \theta_{\gamma}^{k}\right\rangle}, \\
\mathcal{B}_{\gamma \delta \rho}^{v}\left(\mathbf{k}, \mathbf{k}^{\prime}, \mathbf{k}^{\prime \prime}\right) \equiv \frac{\left\langle\mathbf{v}_{\gamma}^{k *} \cdot\left[\left(\mathbf{v}_{\delta}^{k^{\prime}} \cdot \boldsymbol{\nabla}\right) \mathbf{v}_{\rho}^{k^{\prime \prime}}\right]\right\rangle}{\left\langle\mathbf{v}_{\gamma}^{k *} \cdot \mathbf{v}_{\gamma}^{k}+\operatorname{Pr} \theta_{\gamma}^{k *} \theta_{\gamma}^{k}\right\rangle},
\end{gathered}
$$

which can be simplified after employing $\mathbf{V}_{\gamma}^{k}(z)$ $=\left(i \mathbf{k} D W_{\gamma} / k^{2}, W_{\gamma}\right)$, so the operator $\left(\mathbf{v}_{\gamma} \cdot \boldsymbol{\nabla}\right)$ yields $\left(-\mathbf{k} \cdot \mathbf{k}^{\prime} D W_{\gamma} / k_{j}^{2}+W_{\gamma} D\right)$ : 


$$
\begin{aligned}
\mathcal{B}_{\gamma \delta \rho}^{\theta}\left(\mathbf{k}, \mathbf{k}^{\prime}, \mathbf{k}^{\prime \prime}\right)= & \sum_{k^{\prime}, k^{\prime \prime}} \delta_{\mathbf{k}, \mathbf{k}^{\prime}+\mathbf{k}^{\prime \prime}} \int_{0}^{1} \Theta_{\gamma}^{k *}\left(-\frac{\mathbf{k} \cdot \mathbf{k}^{\prime}}{k^{\prime 2}} D W_{\delta}^{k^{\prime}} \Theta_{\rho}^{k^{\prime \prime}}\right. \\
& \left.+W_{\delta}^{k^{\prime}} D \Theta_{\rho}^{k^{\prime \prime}}\right) d z \\
\mathcal{B}_{\gamma \delta \rho}^{v}\left(\mathbf{k}, \mathbf{k}^{\prime}, \mathbf{k}^{\prime \prime}\right)= & \sum_{k^{\prime}, k^{\prime \prime}} \delta_{\mathbf{k}, \mathbf{k}^{\prime}+\mathbf{k}^{\prime \prime}} \int_{0}^{1}\left[-\frac{\left(\mathbf{k}^{\prime} \cdot \mathbf{k}^{\prime \prime}\right)\left(\mathbf{k} \cdot \mathbf{k}^{\prime \prime}\right)}{k^{\prime 2} k^{\prime \prime 2}}\right. \\
& \times D W_{\gamma}^{k *} D W_{\delta}^{k^{\prime}} D W_{\rho}^{k^{\prime \prime}}+\frac{\mathbf{k} \cdot \mathbf{k}^{\prime \prime}}{k^{\prime \prime 2}} \\
& \times D W_{\gamma}^{k *} W_{\delta}^{k^{\prime}} D^{2} W_{\rho}^{k^{\prime \prime}}-\frac{\left(\mathbf{k}^{\prime} \cdot \mathbf{k}^{\prime \prime}\right) k^{2}}{k^{\prime 2}} \\
& \left.\times W_{\gamma}^{k *} D W_{\delta}^{k^{\prime}} W_{\rho}^{k^{\prime \prime}}+W_{\gamma}^{k *} W_{\delta}^{k^{\prime}} D W_{\rho}^{k^{\prime \prime}}\right] d z .
\end{aligned}
$$

Fortunately symmetry and orthogonality relations, as well as suitable approximations, allows us to further reduce these expressions. Therefore we compute separately quadratic and cubic terms.

\section{Quadratic gradient terms}

The coefficients $\alpha_{1}$ and $\alpha_{2}$ are derived from $\alpha\left(\mathbf{k}_{1}, \mathbf{k}_{2}, \mathbf{k}_{3}\right)=\tau_{0} \mathcal{B}_{111}\left(\mathbf{k}_{1}, \mathbf{k}_{2}, \mathbf{k}_{3}\right)$ employing the fact that vertical modes of order higher than the first one do not contribute to the expansion. Therefore around $k=k_{c}$ we can write

$$
\begin{aligned}
\alpha\left(\left|\mathbf{k}^{\prime}+\mathbf{k}^{\prime \prime}\right|^{2}, k^{\prime 2}, k^{\prime \prime 2}\right) \simeq & \alpha\left(k_{c}^{2}, k_{c}^{2}, k_{c}^{2}\right)+\left.\frac{\partial \alpha}{\partial k^{2}}\right|_{k^{2}=k_{c}^{2}}\left(\left|\mathbf{k}^{\prime}+\mathbf{k}^{\prime \prime}\right|^{2}\right. \\
& \left.-k_{c}^{2}\right)+\left.\frac{\partial \alpha}{\partial k^{\prime 2}}\right|_{k^{\prime 2}=k_{c}^{2}}\left(k^{\prime 2}-k_{c}^{2}\right) \\
& +\left.\frac{\partial \alpha}{\partial k^{\prime \prime 2}}\right|_{k^{\prime \prime 2}=k_{c}^{2}}\left(k^{\prime \prime 2}-k_{c}^{2}\right), \quad \text { (B12) }
\end{aligned}
$$

and since $\mathbf{k}=\mathbf{k}_{c}^{i}+\mathbf{q}, \mathbf{k}^{\prime}=\mathbf{k}_{c}^{i+1}+\mathbf{q}^{\prime}, \mathbf{k}^{\prime}=\mathbf{k}_{c}^{i+2}+\mathbf{q}^{\prime \prime}$, we obtain

$$
\begin{aligned}
\int_{k, k^{\prime}, k^{\prime \prime}} \alpha\left(k^{2}, k^{\prime 2}, k^{\prime \prime 2}\right) a_{k^{\prime}}^{i+1} a_{k^{\prime \prime}}^{i+2} e^{i \mathbf{k} \cdot \mathbf{x}} \delta\left(\mathbf{k}-\mathbf{k}^{\prime}-\mathbf{k}^{\prime \prime}\right) d \mathbf{k} d \mathbf{k}^{\prime} d \mathbf{k}^{\prime \prime} \\
=\int_{k^{\prime}, k^{\prime \prime}} \alpha\left(\left|\mathbf{k}^{\prime}+\mathbf{k}^{\prime \prime}\right|^{2}, k^{\prime 2}, k^{\prime \prime 2}\right) a_{k^{\prime}}^{i+1} a_{k^{\prime \prime}}^{i+2} e^{-i \mathbf{k}^{\prime} \cdot \mathbf{x}} e^{-i \mathbf{k}^{\prime \prime} \cdot \mathbf{x}} d \mathbf{k}^{\prime} d \mathbf{k}^{\prime \prime}=e^{i \mathbf{k}_{c} \cdot \mathbf{x}} \alpha_{0} \bar{A}^{i+1} \bar{A}^{i+2}+e^{i \mathbf{k}_{c} \mathbf{x}} \int_{q^{\prime} q^{\prime \prime}} d \mathbf{q}^{\prime} d \mathbf{q}^{\prime \prime} e^{-i \mathbf{q}^{\prime} \cdot \mathbf{x}} e^{-i \mathbf{q}^{\prime \prime} \cdot \mathbf{x}} \\
\quad \times\left[\frac{\partial \alpha}{\partial k^{\prime 2}}\left(2 \mathbf{k}_{c}^{i+1} \cdot \mathbf{q}^{\prime}+q^{\prime 2}\right)+\frac{\partial \alpha}{\partial k^{2}}\left(2 \mathbf{k}_{c}^{i+1} \cdot \mathbf{q}^{\prime}+q^{\prime 2}\right)+\frac{\partial \alpha}{\partial k^{\prime \prime 2}}\left(2 \mathbf{k}_{c}^{i+2} \cdot \mathbf{q}^{\prime \prime}+q^{\prime \prime 2}\right)+\frac{\partial \alpha}{\partial k^{2}}\right. \\
\left.\quad \times\left(2 \mathbf{k}_{c}^{i+2} \cdot \mathbf{q}^{\prime \prime}+q^{\prime \prime 2}+2 \mathbf{k}_{c}^{i+1} \cdot \mathbf{q}^{\prime \prime}+2 \mathbf{q}^{\prime} \cdot \mathbf{q}^{\prime \prime}\right)\right] a_{q^{\prime}}^{i+1} a_{q^{\prime \prime}}^{i+2} \\
=e^{i \mathbf{k}_{c} \cdot \mathbf{x}}\left\{\alpha_{0} \bar{A}^{i+1} \bar{A}^{i+2}+i \beta_{1}\left[\bar{A}^{i+2}\left(\mathbf{n}_{i+1} \cdot \boldsymbol{\nabla}+i \frac{\nabla^{2}}{2 q_{s}}\right) \bar{A}^{i+1}+\bar{A}^{i+1}\left(\mathbf{n}_{i+2} \cdot \boldsymbol{\nabla}+i \frac{\nabla^{2}}{2 q_{s}}\right) \bar{A}^{i+2}\right]+i \beta_{2}\left[\bar{A}^{i+2}\left(\mathbf{n}_{i+2} \cdot \boldsymbol{\nabla}\right) \bar{A}^{i+1}\right.\right. \\
\left.\left.\quad+\bar{A}^{i+1}\left(\mathbf{n}_{i+1} \cdot \boldsymbol{\nabla}\right) \bar{A}^{i+2}+\frac{i}{k_{c}}\left(\boldsymbol{\nabla} \bar{A}^{i+1}\right)\left(\boldsymbol{\nabla} \bar{A}^{i+2}\right)\right]\right\}
\end{aligned}
$$

in which

$$
\begin{gathered}
\alpha_{0} \equiv \alpha\left(k_{c}, k_{c}, k_{c}\right), \\
\beta_{1} \equiv 2 k_{c}\left[\frac{\partial \alpha}{\partial k^{\prime \prime 2}}+\frac{\partial \alpha}{\partial k^{2}}\right]=2 k_{c}\left[\frac{\partial \alpha}{\partial k^{\prime 2}}+\frac{\partial \alpha}{\partial k^{2}}\right]
\end{gathered}
$$

$$
\beta_{2} \equiv 2 k_{c} \frac{\partial \alpha}{\partial k^{2}}
$$

Taking into account that $\hat{\mathbf{n}}_{2}=-\hat{\mathbf{n}}_{3} / 2+\sqrt{3} \hat{\boldsymbol{\tau}}_{3} / 2, \hat{\mathbf{n}}_{3}=-\hat{\mathbf{n}}_{2} / 2$ $-\sqrt{3} \hat{\boldsymbol{\tau}}_{2} / 2$ and neglecting second-order derivatives, we can write the quadratic gradient terms as 


$$
\begin{aligned}
\alpha \rightarrow & \alpha_{0}+i \alpha_{1}\left[\bar{A}^{i+1}\left(\mathbf{n}_{i+2} \cdot \boldsymbol{\nabla}\right) \bar{A}^{i+2}+\bar{A}^{i+2}\left(\mathbf{n}_{i+1} \cdot \boldsymbol{\nabla}\right) \bar{A}^{i+1}\right] \\
& +i \alpha_{2}\left[\bar{A}^{i+1}\left(\boldsymbol{\tau}_{i+2} \cdot \boldsymbol{\nabla}\right) \bar{A}^{i+2}-\bar{A}^{i+2}\left(\boldsymbol{\tau}_{i+1} \cdot \boldsymbol{\nabla}\right) \bar{A}^{i+1}\right],
\end{aligned}
$$

with

$$
\begin{gathered}
\alpha_{1}=2 k_{c}\left[\frac{\partial \alpha}{\partial k^{\prime 2}}+\frac{1}{2} \frac{\partial \alpha}{\partial k^{2}}\right]=\left[\frac{\partial \alpha}{\partial k^{\prime}}+\frac{1}{2} \frac{\partial \alpha}{\partial k}\right], \\
\alpha_{2}=2 k_{c} \frac{\sqrt{3}}{2} \frac{\partial \alpha}{\partial k^{2}}=\frac{\sqrt{3}}{2} \frac{\partial \alpha}{\partial k} .
\end{gathered}
$$

\section{Cubic terms}

The cubic terms in Eq. (B7) are rather complicated but consistently with an amplitude expansion up to the third order we can reduce the integrals to sums over the minimal resonant terms. So we deal with $\left|k_{i}\right|=k_{c}, i=1,2,3$, and amplitudes $A_{k}$, in terms of which the Eq. (B7) becomes

$$
\begin{aligned}
\dot{A}^{k_{1}}(t)= & \sigma^{k_{1}}(k, R) A^{k_{1}}+\mathcal{B}\left(k_{1}, k_{2}, k_{3}\right) \bar{A}^{k_{2}} \bar{A}^{k_{3}} \\
& +\sum_{k^{\prime}, k^{\prime \prime}} \delta_{k_{1}, k^{\prime}+k_{s}} A^{k^{\prime}} \mathcal{B}\left(k_{1}, k^{\prime}, k_{s}\right) \\
& \times \sum_{k^{\prime \prime}, k^{\prime \prime \prime}} \delta_{k_{s}, k^{\prime \prime}+k^{\prime \prime \prime}} \mathcal{C}\left(k_{s}, k^{\prime \prime}, k^{\prime \prime \prime}\right) A^{k^{\prime \prime}} A_{\rho}^{k^{\prime \prime \prime}} .
\end{aligned}
$$

For example, the equation for $A^{k_{1}}$ includes a term $\bar{A}^{k_{2}} \bar{A}^{k_{3}}$, but also the resonant terms

$$
A^{k_{1}} \rightarrow\left\{\begin{array}{l}
\mathbf{k}_{1}+\mathbf{k}_{0} \rightarrow \mathcal{B}\left(\mathbf{k}_{1}, \mathbf{k}_{1}, \mathbf{k}_{0}\right) A^{k_{1}} A^{k_{0}} \\
\mathbf{k}_{7}-\mathbf{k}_{1} \rightarrow \mathcal{B}\left(\mathbf{k}_{1}, \mathbf{k}_{7},-\mathbf{k}_{1}\right) A^{k_{7}} \bar{A}^{k_{1}} \\
\mathbf{k}_{5}+\mathbf{k}_{2} \rightarrow \mathcal{B}\left(\mathbf{k}_{1}, \mathbf{k}_{5}, \mathbf{k}_{2}\right) A^{k_{5}} A^{k_{2}} \\
\mathbf{k}_{3}-\mathbf{k}_{6} \rightarrow \mathcal{B}\left(\mathbf{k}_{1}, \mathbf{k}_{3},-\mathbf{k}_{6}\right) A^{k_{3}} \bar{A}^{k_{6}}
\end{array}\right.
$$

Then the enslaved modes contribute as

$$
\begin{aligned}
& A^{k_{0}} \rightarrow \mathcal{C}\left(k_{0}, k_{1},-k_{1}\right)\left(A^{k_{1}} \bar{A}^{k_{1}}+A^{k_{2}} \bar{A}^{k_{2}},+A^{k_{3}} \bar{A}^{k_{3}}\right), \\
& A^{k_{7}} \rightarrow \mathcal{C}\left(k_{7}, k_{1}, k_{1}\right) A^{k_{1}} A^{k_{1}}, \\
& A^{k_{5}} \rightarrow \mathcal{C}\left(k_{5}, k_{1},-k_{2}\right) A^{k_{1}} \bar{A}^{k_{2}}
\end{aligned}
$$

$$
\bar{A}^{k_{6}} \rightarrow \mathcal{C}\left(k_{6}, k_{1},-k_{3}\right) A^{k_{1}} \bar{A}^{k_{3}} .
$$

This is the general scheme to eliminate horizontal modes. But vertical modes enter also through nonlinearities. For example, for the quadratic terms one gets

$$
\begin{aligned}
\sum_{\delta \rho} & \sum_{k^{\prime}, k^{\prime \prime}} \delta_{k, k^{\prime}+k^{\prime \prime}} \mathcal{B}_{\gamma \delta \rho}\left(k, k^{\prime}, k^{\prime \prime}\right) A_{\delta}^{k^{\prime}} A_{\rho}^{k^{\prime \prime}} \\
= & B_{111}\left(k_{1}, k_{2}, k_{3}\right) \bar{A}_{1}^{k_{2}} \bar{A}_{1}^{k_{3}}+\sum_{\nu} B_{1 \nu 1}\left(k_{1}, k_{2}, k_{3}\right) \\
& \times\left(\bar{A}_{\nu}^{k_{2}} \bar{A}_{1}^{k_{3}}+\bar{A}_{1}^{k_{2}} \bar{A}_{\nu}^{k_{3}}\right),
\end{aligned}
$$

and damped modes $A_{\nu}^{k_{2}}(\nu \neq 1)$ contribute to the cubic term through

$$
A_{\nu}^{k_{2}}=-\frac{\mathcal{B}_{\nu 11}\left(k_{1}, k_{2}, k_{3}\right)}{\sigma_{\nu}} \bar{A}_{1}^{k_{3}} \bar{A}_{1}^{k_{1}}
$$

Taking all these facts into account one arrives to the normal form Eq. (23):

$$
\tau_{0} \partial_{t} A_{1}=\epsilon A_{1}+\alpha \bar{A}_{2} \bar{A}_{3}-g_{1} A_{1}\left|A_{1}\right|^{2}-g_{2} A_{1}\left(\left|A_{2}\right|^{2}+\left|A_{3}\right|^{2}\right),
$$

with the coefficients

$$
\alpha=\tau_{0} \mathcal{B}_{111}\left(k_{1}, k_{2}, k_{3}\right),
$$

$$
\begin{aligned}
g_{1}= & \tau_{0} \sum_{\nu}\left[\frac{\mathcal{B}_{1 \nu 1}\left(k_{1}, k_{7},-k_{1}\right) \mathcal{B}_{\nu 11}\left(k_{7}, k_{1}, k_{1}\right)}{\sigma_{\nu}^{k_{7}}}\right] \\
& \left.+\frac{\mathcal{B}_{1 \nu 1}\left(k_{1}, k_{1}, k_{0}\right) \mathcal{B}_{\nu 11}\left(k_{0}, k_{1},-k_{1}\right)}{\sigma_{\nu}^{k_{0}}}\right], \\
g_{2}= & \tau_{0} \sum_{\nu}\left[\frac{\mathcal{B}_{1 \nu 1}\left(k_{1}, k_{5}, k_{2}\right) \mathcal{B}_{\nu 11}\left(k_{5}, k_{1},-k_{2}\right)}{\sigma_{\nu}^{k_{5}}}\right. \\
& +\frac{\mathcal{B}_{1 \nu 1}\left(k_{1}, k_{1}, k_{0}\right) \mathcal{B}_{\nu 11}\left(k_{0}, k_{1},-k_{1}\right)}{\sigma_{\nu}^{k_{0}}} \\
& \left.+\frac{\mathcal{B}_{1 \nu 1}\left(k_{1}, k_{2}, k_{3}\right) \mathcal{B}_{\nu 11}\left(k_{1}, k_{2}, k_{3}\right)}{\sigma_{\nu}^{k_{1}}}\right] .
\end{aligned}
$$

[1] M. C. Cross and P. C. Hohenberg, Rev. Mod. Phys. 65, 851 (1993).

[2] H. Bénard, Rev. Gen. Sci. Pures Appl. 11, 1261 (1900).

[3] J. P. A. Pearson, J. Fluid Mech. 4, 489 (1958).

[4] C. Pérez-García, B. Echebarria, and M. Bestehorn, Phys. Rev. E 57, 475 (1998).

[5] L. Koschmieder and D. W. Switzer, J. Fluid Mech. 240, 533
(1992).

[6] K. Nitsche and A. Thess, Phys. Rev. E 52, R5772 (1995).

[7] M. F. Schatz, S. J. VanHook, W. D. McCormick, J. B. Swift, and H. L. Swinney, Phys. Rev. Lett. 75, 1938 (1995).

[8] D. A. Nield, J. Fluid Mech. 19, 341 (1964).

[9] J. W. Scanlon and L. A. Segel, J. Fluid Mech. 30, 149 (1967). [10] J. R. Kraska and R. L. Sani, Int. J. Heat Mass Transf. 22, 535 
(1979).

[11] A. Cloot and G. Lebon, J. Fluid Mech. 145, 447 (1984).

[12] M. Bestehorn and C. Pérez-García, Europhys. Lett. 4, 1365 (1987).

[13] J. Bragard and G. Lebon, Europhys. Lett. 21, 831 (1993).

[14] A. Thess and S. Orszag, J. Fluid Mech. 283, 201 (1995).

[15] P. M. Parmentier, V. C. Regnier, G. Lebon, and J. C. Legros, Phys. Rev. E 54, 411 (1996).

[16] M. Bestehorn, Phys. Rev. E 48, 3622 (1993).

[17] J. Bragard and M. G. Velarde, J. Fluid Mech. 368, 165 (1998).

[18] A. A. Golovin, A. A. Nepomnyashchy, and L. M. Pismen, J. Fluid Mech. 341, 317 (1997).

[19] J. Lauzeral, S. Metens, and D. Walgraef, Europhys. Lett. 24, 707 (1993).

[20] M. M. Sushchik and L. S. Tsimring, Physica D 74, 90 (1994).

[21] R. Hoyle, Appl. Math. Lett. 9, 81 (1995).

[22] S. J. VanHook, M. F. Schatz, W. D. McCormick, J. B. Swift, and H. L. Swinney, Phys. Rev. Lett. 75, 4397 (1995).

[23] S. H. Davis and G. M. Homsy, J. Fluid Mech. 98, 3 (1980).

[24] M. Schatz (private communication).

[25] H. F. Golubitsky, J. W. Swift, and E. Knobloch, Physica D 10, 249 (1984).

[26] P. Manneville, Structures Dissipatives, Chaos et Turbulence (Aléa Saclay, Paris, 1991).

[27] H. Brand, Prog. Theor. Phys. Suppl. 99, 442 (1989).
[28] G. Gunaratne, Q. Ouyang, and H. L. Swinney, Phys. Rev. E 50, 2802 (1994).

[29] E. A. Kuznetsov, A. A. Nepomnyashchy, and L. M. Pismen, Phys. Lett. A 205, 261 (1995).

[30] E. L. Koschmieder and M. I. Biggerstaff, J. Fluid Mech. 167, 49 (1986).

[31] P. C. Dauby, G. Lebon, P. Colinet, and J. C. Legros, Q. J. Mech. Appl. Math. 46, 683 (1993).

[32] A. Thess and M. Bestehorn, Phys. Rev. E 52, 6358 (1995).

[33] S. Zaleski, Thèse 3ème cycle, Université de Paris VI 1980 (unpublished).

[34] R. Kuske and P. Milewski, Eur. J. Appl. Math. 10, 157 (1999).

[35] B. Echebarria and C. Pérez-García, Europhys. Lett. 43, 35 (1998).

[36] A. E. Nuz, A. A. Nepomnyashchy, A. A. Golovin, A. A. Hari, and L. M. Pismen, Physica D 135, 233 (2000).

[37] K. Eckert, M. Bestehorn, and A. Thess, J. Fluid Mech. 356, 155 (1998).

[38] M. F. Schatz, S. J. VanHook, W. D. McCormick, J. B. Swift, and H. L. Swinney, Phys. Fluids 11, 2577 (1999).

[39] M. Bestehorn, Phys. Rev. Lett. 76, 46 (1996).

[40] V. Regnier, P. C. Dauby, P. Parmentier, and G. Lebon, Phys. Rev. E 55, 6860 (1997).

[41] A. Engel and J.B. Swift, Phys. Rev. E 62, 6250 (2000). 